\title{
Update monotone preference rules
}

Citation for published version (APA):

Can, B., \& Storcken, A. J. A. (2011). Update monotone preference rules. METEOR, Maastricht University School of Business and Economics. METEOR Research Memorandum No. 048 https://doi.org/10.26481/umamet.2011048

Document status and date:

Published: 01/01/2011

DOI:

10.26481/umamet.2011048

Document Version:

Publisher's PDF, also known as Version of record

\section{Please check the document version of this publication:}

- A submitted manuscript is the version of the article upon submission and before peer-review. There can be important differences between the submitted version and the official published version of record.

People interested in the research are advised to contact the author for the final version of the publication, or visit the DOI to the publisher's website.

- The final author version and the galley proof are versions of the publication after peer review.

- The final published version features the final layout of the paper including the volume, issue and page numbers.

Link to publication

\footnotetext{
General rights rights.

- You may freely distribute the URL identifying the publication in the public portal. please follow below link for the End User Agreement:

www.umlib.nl/taverne-license

Take down policy

If you believe that this document breaches copyright please contact us at:

repository@maastrichtuniversity.nl

providing details and we will investigate your claim.
}

Copyright and moral rights for the publications made accessible in the public portal are retained by the authors and/or other copyright owners and it is a condition of accessing publications that users recognise and abide by the legal requirements associated with these

- Users may download and print one copy of any publication from the public portal for the purpose of private study or research.

- You may not further distribute the material or use it for any profit-making activity or commercial gain

If the publication is distributed under the terms of Article $25 \mathrm{fa}$ of the Dutch Copyright Act, indicated by the "Taverne" license above, 


\section{Maastricht University}

Burak Can, Ton Storcken

Update Monotone Preference Rules

$\mathrm{RM} / 11 / 048$

\section{METEOR}

Maastricht University School of Business and Economics

Maastricht Research School of Economics

of Technology and Organization

P.O. Box 616

NL - 6200 MD Maastricht

The Netherlands 


\title{
Update Monotone Preference Rules
}

\author{
Burak Can* Ton Storcken ${ }^{\dagger}$
}

October 2011

\begin{abstract}
Collective decisions are modeled by preference correspondences (rules). In particular, we focus on a new condition: "update monotonicity" for preference rules. Although many so-called impossibility theorems for the choice rules are based on -or related to- monotonicity conditions, this appealing condition is satisfied by several non-trivial preference rules. In fact, in case of pairwise, Pareto optimal, neutral, and consistent rules; the Kemeny-Young rule is singled out by this condition. In case of convex valued, Pareto optimal, neutral and replication invariant rules; strong update monotonicity implies that the rule equals the union of preferences which extend all preference pairs unanimously agreed upon by $k$ agents, where $k$ is related to the number of alternatives and agents. In both cases, it therewith provides a characterization of these rules.
\end{abstract}

JEL Classification: D63, D71, D72

Keywords: Update monotonicity, Preferences, Aggregation, Kemeny-Young Method

\section{Introduction}

Consider a collective decision making problem where, by means of a preference correspondence ${ }^{1}$, individual strict preferences, i.e. complete, transitive, and antisymmetric binary relations over a set of alternatives, are aggregated into a set of strict preferences. For instance, to appoint a new dean, a committee of department representatives collectively ranks three candidates: $A($ lice $), B($ ill $)$ and $C$ (aroline). Let the outcome be such that $A$ is ranked the best, $B$ is second best and $C$ is ranked the lowest. Suppose now that one of the representatives, preferring $C$ above $B$ and both these above $A$, is substituted by another member of that department with preference $A$ above $C$ above $B$. Clearly, the latter preference agrees on more ordered pairs of the outcome than the former does and it only differs with the outcome on pairs where the former differs with the outcome. So to speak, the latter preference is an "update" of the former towards the outcome. The question raised here is whether in the new hypothetical situation the outcome would still be $A$ above $B$ above $C$.

Take a (preference) profile, i.e. a combination of individual preferences, and a possible outcome of a preference correspondence. By updating, we refer to transformations of the profile such that the

* Corresponding author: Department of Economics, School of Business and Economics, Maastricht University, P.O. Box 616, 6200 MD Maastricht, The Netherlands; E-mail: b.can@maastrichtuniversity.nl. Authors are grateful to Bettina Klaus, Kristof Bosmans and Tuba Korkmaz for their comments on earlier drafts. The support of Netherlands Organisation for Scientific Research (NWO) under the grant with project nr. 400-09-354, is gratefully acknowledged.

${ }^{\dagger}$ Department of Quantitative Economics, Maastricht University, P.O. Box 616, NL 6200 MD Maastricht, E-mail: t.storcken@ke.unimaas.nl, Phone number: +31 43 3883728, Fax number:+31 433884874

${ }^{1}$ Set valuedness of an aggregation rule may reflect the possible outcomes of neutral tie-breaking. 
individual preferences change only in parts where they differ from the given outcome. Therewith, these transformations yield profiles which are more similar to the outcome and which are, in a sense, updates towards it. A preference correspondence is said to be update monotone if the outcome is still chosen at such a transformation. Strong update monotonicity, moreover, requires that the outcomes at the updated profile form a subset of the previous set of outcomes.

For collective choice functions and choice correspondences (for simplicity referred to as choice rules) various monotonicity conditions have been studied intensively. Well-known impossibility theorems, such as in Muller and Satterthwaite (1977), express that only trivial choice rules such as dictatorial rules or constant rules are monotone. Therefore, imposing this type of condition to choice rules can seem too restrictive. Furthermore, when defining a monotonicity condition for a choice rule, the description of a preference change in favour of an alternative is not completely free of contamination. For instance, extending the lower contour set of a winning alternative, as in Maskin monotonicity, assumes that these choice rules should not depend on how these lower contour sets are ordered individually. In the case of preference rules and update monotonicity, however, demanding that individual preferences can only change as far as they differ from the outcome is at least intuitively more sound. Furthermore monotonicity conditions have not yet been analyzed for preference rules.

In comparison to these monotonicity conditions for choice rules, we draw the conclusion that the ones presented here for preference rules seem less restrictive. Indeed, imposing update monotonicity on preference rules along with other conditions, which are satisfied by many well-known preference rules, yields characterizations of "Kemeny-Young" and "super majority" preference rules. The main reason for this is that compared to choice rules, the framework of preference rules allows for more detail in expressing monotonicity.

In the choice function framework it is also well-known that the aforementioned impossibility theorems based on monotonicity conditions correspond to some impossibility theorems based on strategy-proofness conditions. In the preference rule framework, Bossert and Storcken (1992) showed that there exists no coalitional strategy-proof welfare function, i.e., single valued preference correspondence, which is in addition nonimposed and weak extrema independent. However, for welfare functions update monotonicity is compatible with these two latter conditions as is discussed in Section 5.

Update monotonicity is discussed for two disjoint cases; the first in which we assume that preference rules are convex valued and the second where this is not required. In the first case we determine the class of Pareto optimal, neutral, replication invariant, convex valued and strongly update monotone preference rules. It consists of super majority rules. At these rules, depending on the number of alternatives and the number of individuals, a number $k$ close to the number of agents $n$ is fixed. Now these correspondences assign all preferences which extend all ordered pairs unanimously agreed upon by any set of at least $k$ individuals. This result characterizes super majority rules as the only preference rules that satisfy the five conditions mentioned above. Pareto optimality, neutrality and replication invariance are conditions met by many well-known preference correspondences. Whenever set valued outcomes stem from tie breaking indifferences, the convexity requirement on these outcome sets means that indifferences are broken in every possible way. Many well-known preference correspondences are convex valued. The characterization of super majority rules therefore implies that many well-known rules, such as the Borda (1784) and the Copeland (1951) rule, are not strongly update monotone. Moreover, in deducing this characterization of super majority rules we have the following result based on Pareto optimality, anonymity, neutrality, convex 
valuedness and update monotonicity only. Consider profiles where the set of agents is partitioned into two groups such that all members in each group report the same preference and the two reported preferences differ on precisely one consecutively ordered pair of alternatives only. At these profiles, which in fact resemble a decision situation on two alternatives, the outcome is both of these preferences if no group has more than $\frac{m-1}{m} n$ agents, where $m$ is the number of alternatives and $n$ the number of agents. So, in case of 3 alternatives a $\frac{2}{3}$ majority is not decisive in such a situation. This result shows that many well-known rules do not satisfy update monotonicity. In the setting of convex valued preference rules, the strong version of update monotonicity is necessary for the characterization of super majority rules. The logical independence of these characterizing conditions is discussed by examples; and furthermore, the necessity of the stronger version of update monotonicity is shown.

Next, consider the situation of possibly non-convex valued outcomes. The Kemeny-Young preference correspondence (simply the Kemeny rule), assigning those strict preferences to a given profile which minimize the sum of Kemeny distance to all of the individual preferences in the profile, is update monotone. It is, however, not convex valued. For instance in case of three alternatives at a Condorcet profile, unlike many preference correspondences having all six strict preferences in the outcome, it only assigns the three preferences reported in the profile. The Kemeny rule is the only rule which is Pareto optimal, consistent, pairwise, neutral and update monotone. The former four conditions are well-known and satisfied by various preference rules. Comparing the two characterizations, we see a substitution of convexity by pairwiseness and a trade off between a strengthening of replication invariance to consistency and a weakening of update monotonicity.

Young and Levenglick (1978) characterized the Kemeny rule by neutrality, consistency and the Condorcet condition. A major step in their proof is that given a profile, outcomes assigned by rules satisfying these three conditions are determined by a pairwise difference matrix which records the numerical differences of pairwise comparisons in its cell. That is, if two profiles yield the same pairwise difference matrix, then the outcomes at these two profiles are equal. Herewith such rules are pairwise. A similar result can be found in Lemma 11. From that point on, because of the differences in the characterizing conditions, the line of arguments and, hence, the proofs differ completely. In addition to the logical independence of the five conditions we employ, we also show their logical equivalence to those used by Young and Levenglick

The paper proceeds as follows: in Section 2, we provide some notation for our model and briefly discuss certain properties including update monotonicity, and provide examples of some rules to which we refer in the later sections. Section 3 is devoted to the class of convex valued rules. An intuition is provided on why many convex valued rules are not update monotone. Thereafter, we provide a characterization of the family of super majority correspondences as indicated above. Section 4 discusses preference correspondences where convex valuedness is dropped and here we provide a new characterization of the Kemeny rule based on update monotonicity. In Section 5, we discuss the findings, such as the logical independence of the characterizing conditions and their logical equivalence to the conditions of Young and Levenglick. Furthermore, some weakening and strengthening of update monotonicity are formulated and discussed briefly. Finally, we show that in the social welfare setting of Bossert and Storcken (1992), update monotonicity is less demanding than strategy-proofness. 


\section{The Model}

\subsection{Basic Notation}

Let $A$ be a finite set of alternatives with cardinality $\# A=m>1$. Preferences are taken to be linear orders over the set of alternatives $A$. Let $\mathbb{L}$ denote the set of all preferences over $A$. Let $\mathcal{N}$ be a countable infinite set of potential agents. For non-empty and finite subsets $N$ of $\mathcal{N}$ with cardinality $n, \mathbb{L}^{N}$ denotes the set of all preference profiles $p$, i.e., an $n$-dimensional vector of preferences where its $i^{\text {th }}$ component $p(i)$ refers to individual $i$ 's preference. The restriction of profile $p$ to a subset of agents, say $S$, is denoted by $\left.p\right|_{S}$. For situations where two disjoint sets of agents $N, N^{\prime} \in \mathcal{N}$, with preference profiles $p \in \mathbb{L}^{N}$, and $q \in \mathbb{L}^{N^{\prime}}$ over the same set of alternatives are united, we interpret $(p, q)$ as the union ${ }^{2}$ of these two profiles, i.e., $(p, q) \in \mathbb{L}^{N \cup N^{\prime}}$ with $(p, q)(i)=p(i)$ if $i \in N$ and $(p, q)(i)=q(i)$ if $i \in N^{\prime}$. Furthermore, for a coalition $S$, a nonempty subset of $N$, and a linear order $R$, let $R^{S}$ denote the profile $p$ where $p(i)=R$ for all $i$ in $S$. So, $R^{N}$ denotes the the unanimous profile in which all agents in $N$ have preference $R$. A preference correspondence or simply a rule $\varphi$, is a function such that for every finite and non-empty set $N \subset \mathcal{N}, \varphi$ assigns a nonempty subset $\varphi(p)$ of $\mathbb{L}$, to each preference profile $p$ in $\mathbb{L}^{N}$.

Let $R$ be a linear order on $A$. Consider three different alternatives $a, b, c \in A$. Note that because of anti-symmetry of $R,(a, b) \in R$ means that $a$ is strictly preferred to $b$, which hereafter will be denoted by ".a.b. $=R$ ". The case where $a$ and $b$ are consecutive at $R$, i.e., there is no alternative $c$ that is ordered in between $a$ and $b$, is denoted by $. a b .=R$. Notations like $. a . b . c .=R$ and $. a b . c .=R$ have the obvious interpretation. Furthermore $a \ldots=R$ means that alternative $a$ is ordered best at $R$ and likewise $\ldots b=R$ means that alternative $b$ is ordered worst at $R$. Let $P$ be a partial order, i.e. an anti-symmetric, reflexive, and transitive binary relation on $A$. Then, $\mathbb{L}_{P}=\{R \in \mathbb{L}: P \subseteq R\}$ denotes the set of linear extensions of $P$. To save parenthesis, we write $\mathbb{L}_{a b}$ instead of $\mathbb{L}_{\{(a, b)\}}$. Let $a_{1} a_{2} \ldots a_{l-1} a_{l} a_{l+1} \ldots a_{m}=R$ and $a_{1} a_{2} \ldots a_{l-1} a_{l+1} a_{l} \ldots a_{m}=\bar{R}$. Then we say $R$ (respectively $\bar{R}$ ) is an elementary change of $\bar{R}(R)$ in pair $a_{l} a_{l+1}\left(a_{l+1} a_{l}\right)$ or $R$ and $\bar{R}$ form an elementary change (in $a_{l}$ and $\left.a_{l+1}\right)$. Furthermore this elementary change is in position $l$, i.e., the $l^{t h}$ and $(l+1)^{t h}$ alternatives are swapped.

Given a nonempty subset of alternatives $B \subseteq A$, let $\left.R\right|_{B}$ denote the restriction of an order $R$ to $B$, i.e., $\left.R\right|_{B}=\{(x, y) \in R: x, y \in B\}$. In a similar way let $\left.p\right|_{B}$ be the restriction of a profile $p$ to $B$, i.e., $\left(\left.p\right|_{B}\right)(i)=\left.p(i)\right|_{B}$ for every agent $i$ in $N$.

For preferences $R^{1}, R^{2}$, and $R^{3}$, we say $R^{3}$ is between ${ }^{3} R^{1}$ and $R^{2}$ if and only if $R^{1} \cap R^{2} \subseteq R^{3}$. The Kemeny distance (Kemeny, 1959; Kemeny and Snell, 1962) for two linear orders $R^{1}$ and $R^{2}$ is defined by $\delta\left(R^{1}, R^{2}\right)=\frac{1}{2} \#\left(\left(R^{1}-R^{2}\right) \cup\left(R^{2}-R^{1}\right)\right)=\#\left(R^{1}-R^{2}\right)$, i.e., half of the cardinality of the symmetric difference of $R^{1}$ and $R^{2}$. Note that $\delta$ is a distance function and hence satisfies the triangular inequality. Furthermore, for any three linear orders; $R^{1}, R^{2}$ and $R^{3}$, we have:

$$
\delta\left(R^{1}, R^{3}\right)+\delta\left(R^{3}, R^{2}\right)=\delta\left(R^{1}, R^{2}\right) \text { if and only if } R^{3} \text { is between } R^{1} \text { and } R^{2} .
$$

Finally let $\mathbb{S}$ be a nonempty subset of $\mathbb{L}$. $\mathbb{S}$ is convex if for all $R^{1}$ and $R^{2}$ in $\mathbb{S}$, and for all $R^{3}$ in $\mathbb{L}$, $R^{3}$ is also in $\mathbb{S}$ if $R^{3}$ is between $R^{1}$ and $R^{2}$. In Bogart (1973) there is a discussion of betweenness and the concept of convexity. In Storcken (2008), the following characterization can be found:

\footnotetext{
${ }^{2}$ We abuse notation by dropping parentheses whenever it is clear that we refer to the union of these two set of agents.

${ }^{3}$ For general relations also the condition $R^{3} \subseteq R^{1} \cup R^{2}$ is needed. In case of linear orders, this, however, is equivalent to $R^{1} \cap R^{2} \subseteq R^{3}$.
} 
Proposition 1 Let $\mathbb{S}$ be a subset of $\mathbb{L}$. Then the following two are equivalent $(\boldsymbol{i}) \mathbb{S}$ is convex,(ii)

There is a partial order $P$ such that $\mathbb{L}_{P}=\mathbb{S}$.

\subsection{Properties of Rules}

Next we discuss some properties for preference correspondences that we will use in the following sections.

Single valuedness: A rule is single valued if the outcome at every profile is a singleton, i.e. is a set of precisely one linear order. Clearly, this condition means that the rule is a function. In the literature these functions are also known as welfare functions.

Pareto Optimality: The Pareto condition requires that all preferences assigned to a profile are Pareto optimal. Formally: $\varphi(p) \subseteq \mathbb{L}_{\cap\{p(i): i \in N\}}$ for all profiles $p$ in $\mathbb{L}^{N}$.

Anonymity: Anonymity requires that all individuals are treated equally; hence, renaming them should not change the outcome, i.e. $\varphi(p)=\varphi(p \circ \pi)$ for all profiles $p$ in $\mathbb{L}^{N}$ and all permutations $\pi$ of $N$, where $p \circ \pi$ is the profile such that $p \circ \pi(i)=p(\pi(i))$ for all $i \in N$.

Neutrality: A rule is said to be neutral whenever it treats alternatives in a neutral way: $\tau(\varphi(p))=$ $\varphi(\tau p)$ for all profiles $p$ in $\mathbb{L}^{N}$ and all permutation $\tau$ of $A$, where $\tau$ extends to any relation $R$ on $A$ by $\tau(R)=\{(\tau(a), \tau(b)):(a, b) \in R\}, \tau(\mathbb{S})=\{\tau(R): R \in \mathbb{S}\}$ denotes the complete image of a set $\mathbb{S}$ under $\tau$ and $\tau p$ is defined for an individual $i$ by $(\tau p)(i)=\tau(p(i))$.

Replication Invariance: Replication invariance means that the outcome of a rule is the same before and after the replication of a profile: $\varphi(p)=\varphi\left(p^{1}, p^{2}, \ldots, p^{k}\right)$ for all profiles $p, p^{1}, p^{2}, \ldots$ and $p^{k}$ in respectively $\mathbb{L}^{N}, \mathbb{L}^{N_{1}}, \mathbb{L}^{N_{2}} \ldots$ and $\mathbb{L}^{N_{k}}$ such that $N, N_{1}, N_{2} \ldots N_{k}$ are all pairwise disjoint and there are bijections $\sigma_{t}$ from $N$ to $N_{t}$ for all $t \in\{1,2, \ldots k\}$ such that $p(i)=p^{t}\left(\sigma_{t}(i)\right)$ for all $i$ in $N$ and $\left(p^{1}, p^{2}, \ldots, p^{k}\right)$ is the profile say $q$ on $N_{1} \cup N_{2} \cup \ldots N_{k}$, with $q(i)=p^{t}(i)$ for all $i \in N_{t}$.

Consistency: Consistency requires that the outcome assigned to a profile, which is composed of two disjoint sets of individuals that are merged, equals the intersection of the outcomes assigned to the profiles of these sets of individuals separately, whenever this intersection is non-empty. Formally: $\varphi(p) \cap \varphi(q)=\varphi(p, q)$ for all profiles $p$ and $q$ in respectively $\mathbb{L}^{N}$ and $\mathbb{L}^{M}$ where $N$ and $M$ are disjoint and $\varphi(p) \cap \varphi(q) \neq \emptyset$.

Pairwiseness: A rule is pairwise if the outcomes at two profiles are equal whenever every pairwise comparison in a profile is numerically equal to that in the other profile. Formally: $\varphi(p)=\varphi(q)$ for all profiles $p$ and $q$ in $\mathbb{L}^{N}$ such that $M(p)=M(q)$, where $M(p)$ is a $m$ by $m$ matrix such that for alternatives $a$ and $b$, cell $(a, b)$ is defined by the number of agents preferring $a$ to $b$ :

$$
\begin{aligned}
M(p)_{(a, b)} & =\#\{i \in N:(a, b) \in p(i)\} \text { if } a \neq b \\
& =0 \text { if } a=b .
\end{aligned}
$$

Convex valuedness: A rule satisfies convex valuedness if it assigns to each profile a convex set of linear orders: $\varphi(p)$ is convex for all profiles $p$ in $\mathbb{L}^{N}$.

Remark 1 Note that replication invariance implies anonymity, and consistency implies replication invariance.

Below we provide some examples of convex valued rules:

Example 1 (Score rules) Let $\vec{s}=\left(s_{1}, \ldots s_{m}\right)$ be the score vector over the set of alternatives such that $s_{1} \geq \ldots \geq s_{m}$ and $s_{1}>s_{m}$. Let rank $(a, p(i))=\#\{b \in A: . b . a .=p(i)$ or $a=b\}$, i.e. 
the rank of alternative $a$ in $i^{\text {th }}$ preference $p(i)$ equals the the number of alternatives preferred or indifferent to $a$. For each alternative $a \in A$, let score $(\vec{s}, a, p)=\sum_{i \in N} s_{\text {rank }(a, p(i))}$ be the sum of scores of alternative a in each individual preference $p(i)$ in the profile $p$. Consider the partial order $P_{\vec{s}}(p)=(a, b): \operatorname{score}(\vec{s}, a, p)>$ score $(\vec{s}, b, p)$ or $\left.a=b\right\}$. The score rule $\varphi \vec{s}$ induced by the score vector $\vec{s}$ equals is defined for profile $p$ by

$$
\varphi \vec{s}(p)=\mathbb{L}_{P_{\vec{s}}(p)},
$$

where $\mathbb{L}_{P_{\vec{s}}(p)}=\left\{R \in \mathbb{L}: P_{\vec{s}}(p) \subseteq R\right\}$.

Example 2 (Copeland rule) Let $s_{C}(a, p)$ denote the Copeland score of alternative a in preference profile $p$ which is the number of alternatives a beats in pairwise comparisons, i.e., $\quad \#\{b \in A \backslash\{a\}: \#\{i \in N:$ a.b. $=p(i)\}>n / 2\}$. Consider the partial order $P_{c}(p)=$ $\left\{(a, b): s_{C}(a, p)>s_{C}(b, p)\right.$ or $\left.a=b\right\}$. The Copeland rule $\varphi_{C}$ is defined for profile $p$ by

$$
\varphi_{C}(p)=\mathbb{L}_{P_{c}(p)},
$$

where $\mathbb{L}_{P_{c}(p)}=\left\{R \in \mathbb{L}: P_{c}(p) \subseteq R\right\}$.

Note that by Proposition 1, score rules and the Copeland rule, as defined in the examples above, satisfy convex valuedness.

Example 3 (Pareto rule) For each profile $p$, the Pareto rule $\varphi_{\text {Par }}$ is defined $\varphi_{\text {Par }}(p)=$ $\mathbb{L}_{\cap\{p(i): i \in N\}}$.

Example 4 ( $k$-majority rule) Let $A$ consist of $m$ alternatives and $N$ be a set of $n$ agents. Let $k$ be a positive integer such that $\frac{m-1}{m} n<k \leq n$. For any profile $p$ define $k$-majority-relation $(p)=$ $\{(x, y) \in A \times A$ : there are at least $k$ agents $i$ such that .x.y. $=p(i)\}$ and define the $k$-majority

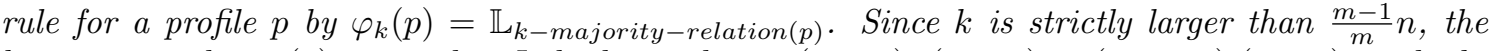
$k$-majority-relation $(p)$ is acyclic. Indeed a cycle say $\left(a_{1}, a_{2}\right),\left(a_{2}, a_{3}\right), \ldots,\left(a_{l-1}, a_{l}\right),\left(a_{l}, a_{1}\right)$ in the $k$ majority-relation $(p)$ would imply the existence of subsets $S^{1}, S^{2}, \ldots, S^{l-1}, S^{l}$ of $N$ such that $\# S^{j} \geq k$ and $S^{j}=\left\{i \in N: a_{j} . a_{j+1} .=p(i)\right\}$ for $j \in\{1, \ldots, l\}$ and where further $a_{l+1}$ is set equal to $a_{1}$. As preferences $p(i)$ are transitive and therefore in particular acyclic it follows that $\cap\left\{S^{j}: j \in\right.$ $\{1, \ldots, l\}\}=\emptyset$. But as $\# S^{j} \geq k>\frac{m-1}{m} n \geq \frac{l-1}{l} n$, because $l \leq m$, this result would contradict Proposition 4 (see the Appendix). Therefore $\mathbb{L}_{k-m a j o r i t y-r e l a t i o n(p)}$ is non-empty and $\varphi_{k}$ is welldefined. Rule $\varphi_{k}$ is convex valued by definition and it is obvious that it is neutral, Pareto optimal

and anonymous. Note that in case $k=n$ rule $\varphi_{k}$ equals the Pareto rule, $\varphi_{P a r}$. Further note that $\mathbb{L}_{k-\text { majority-relation }(p)}=\{R \in \mathbb{L}: \cap\{p(i): i \in S\} \subseteq R$ for all $\# S \geq k\}$.

\section{$2.3 \quad$ Update Monotonicity}

Consider profile $p$ and linear order $R$ an outcome of some rule at that profile. We call profile $q$ an update of $p$ towards $R$ if $p(i) \cap R \subseteq q(i)$ for all agents $i \in N$. So, for all agents $i$ this means that $R$ and $q(i)$ have at least in common what $p(i)$ and $R$ have. Or to put it differently, for all agents $i$, preference $q(i)$ only differs from $R$ on pairs where $p(i)$ differs from $R$. Loosely speaking, this boils down to $q(i)$ and $R$ having more pairs in common than $p(i)$ and $R$ have. Note that in that case $q(i)$ is between $p(i)$ and $R$ for all agents $i$. Based on this update, the following notions of monotonicity are defined. 
(Update) Monotonicity: A rule $\varphi$ is update monotone if for all $p$ in $\mathbb{L}^{N}$, for all $R \in \varphi(p)$, and for all updates $q$ of $p$ towards $R$,

$$
R \in \varphi(q) .
$$

Furthermore, $\varphi$ is strongly update monotone if it is update monotone and $\varphi(q) \subseteq \varphi(p)$ for all such outcomes $R$ in $\varphi(p)$ and profiles $q$.

From this point, on we use the word monotonicity instead of update monotonicity whenever it is clear that we mean the latter. Note that betweenness implies that the Kemeny distance between the collective preference $R$ and each individual preference is not increased from profile $p$ to profile $q$. Indeed monotonicity can be defined in a stronger way by only demanding that these distances do not increase and therewith dropping the betweenness condition. In Section 5 this is discussed in more detail.

Because the set of profiles is connected by elementary changes we have the following immediate result.

Proposition 2 A rule $\varphi$ is monotone if $\mathbb{L}_{a b} \cap \varphi(p) \subseteq \varphi(q)$ for all profiles $p, q \in \mathbb{L}^{N}$, such that $\mathbb{L}_{a b} \cap \varphi(p) \neq \emptyset$ and there are agents $j \in N$ and alternatives $a, b \in N$ with $\left.p\right|_{N-\{j\}}=\left.q\right|_{N-\{j\}}$, $. a b .=q(j)$, .ba. $=p(j)$ and $q(j)$ is an elementary change of $p(j)$ in pair ab. Furthermore, $\varphi$ is strongly monotone if and only if it is monotone and $\varphi(q) \subseteq \varphi(p)$ for such $p, q$ and alternatives a and $b$.

Remark 2 As the empty set is contained in any set, it follows immediately that $\varphi$ is monotone if and only if $\mathbb{L}_{a b} \cap \varphi(p) \subseteq \varphi(q)$ for all profiles $p, q \in \mathbb{L}^{N}$, such that there are agents $j \in N$ and alternatives $a, b \in N$ with $\left.p\right|_{N-\{j\}}=\left.q\right|_{N-\{j\}}$, .ab. $=q(j), . b a .=p(j)$ and $q(j)$ is an elementary change of $p(j)$ in pair ab. The requirement $\mathbb{L}_{a b} \cap \varphi(p)$ being non-empty is important, though more for strong monotonicity. Actually, monotonicity implies both $\mathbb{L}_{a b} \cap \varphi(p) \subseteq \varphi(q)$ and $\mathbb{L}_{b a} \cap \varphi(q) \subseteq \varphi(p)$. If $\mathbb{L}_{a b} \cap \varphi(p)$ is non-empty, then $\varphi(q) \subseteq \varphi(p)$. If in addition $\mathbb{L}_{b a} \cap \varphi(q)=\emptyset$, a situation which holds for the Kemeny rule, we have $\mathbb{L}_{a b} \cap \varphi(p) \subseteq \varphi(q), \varphi(q) \subseteq \varphi(p)$ and $\varphi(q) \subseteq \mathbb{L}_{a b}$. Hence, then $\mathbb{L}_{a b} \cap \varphi(p)=\varphi(q)$. Note also that a rule $\varphi$ is strongly monotone if for all alternative $a$ and $b$ and all profiles like $p$ and $q$ we have $\mathbb{L}_{a b} \cap \varphi(p)=\varphi(q)$ whenever $\mathbb{L}_{a b} \cap \varphi(p)$ is non-empty.

\section{Convex Valued Rules}

As pointed out in the introduction and in the examples of the previous section, many preferences rules, such as score rules, the Kramer rule and the Copeland rule, are convex valued. In this section, we discuss the consequences of strong monotonicity under the assumption of convex valued outcomes. To structure this discussion further, we only consider rules satisfying the following basic conditions: Pareto optimality, neutrality and replication invariance. The latter condition is a strengthening of anonymity and links different sets of agents. These five conditions together characterize the class of super majority rules. To each profile, these rules assign all linear orders that extend the ordered pairs for which there are at least $k$ agents who unanimously agree upon them. The number $k$ is chosen in such a way that the pairs for the possibly different sets of $k$ agents cannot form a cyclical decision. Therefore $k$ depends on the number of alternatives and the number of agents.

To illustrate how convex valuedness and monotonicity under the other three conditions bring about this characterization, consider the outcome of an arbitrary rule $\varphi$ satisfying these conditions 
at a standard Condorcet profile $p$ for three agents 1,2 , and 3 and three alternatives $a, b$, and $c$ defined by:

$$
\begin{aligned}
& p(1)=a b c \\
& p(2)=b c a \\
& p(3)=c a b .
\end{aligned}
$$

Here preferences are denoted by their representation. So, $a b c$ denotes the linear order at which $a$ is the most preferred, $b$ the second most and $c$ is considered the worst. Assume $a b c \in \varphi(p)$. Then by neutrality $b c a, c a b \in \varphi(p)$. Now $a b c, b c a, c a b \in \varphi(p)$ and convex valuedness imply $\varphi(p)=\mathbb{L}$. Assume $a c b \in \varphi(p)$. Then by neutrality $b a c, c b a \in \varphi(p)$. Now $a c b, b a c, c b a \in \varphi(p)$ and convex valuedness again imply $\varphi(p)=\mathbb{L}$. This means that such rules will assign the set of all possible preferences to the Condorcet profile $p$. In particular bac $\in \varphi(p)$. Next, consider the preference profile $q$ defined by

$$
\begin{aligned}
& q(1)=a b c=p(1) \\
& q(2)=b a c \\
& q(3)=a b c=p(1)
\end{aligned}
$$

Note that $q$ is an update of $p$ towards both $b a c$ and $a b c$. Because of monotonicity, $\varphi$ therefore assigns $a b c$ and $b a c$ to profile $q$, which by Pareto optimality then implies that $\varphi(q)=\{a b c, b a c\}$. Note that $b a c$ is among the outcome at profile $p$ where only one-third of the population preferences is in line with this ranking and two-third is opposing it. Many commonly used rules, however, take only the two third majority point of view $a b c$ at this profile $q$. This means that these rules do not satisfy all five conditions. In fact they defect on monotonicity.

The question now is "how large a coalition, say $S$, should be" in order to ensure that $\varphi$ only assigns the opinion of this coalition $S$ and therewith ignores that of the minority $N-S$. The example above clarifies that in case of three alternatives $S$ should consist of strictly more than two third of the agents present. Indeed, this makes sense for $k$-majority rules, because in the three alternatives three agents case letting $k=2$ would yield a cycle since two agent coalition $\{1,2\}$ is unanimous on $b c$, coalition $\{2,3\}$ on $c a$ and coalition $\{1,3\}$ on $a b$. But this cycle cannot be extended to a linear order.

Unless stated otherwise, for the remainder of this section, we switch to the stronger version of monotonicity. This is because we were not able to determine the class of Pareto optimal, neutral, replication invariant, convex valued and monotone rules. It is clear that this class is larger than that of super majority rules. For instance, biased super majority rules, introduced in the discussion section, are not strongly monotone but they are monotone.

The class of Pareto optimal, neutral, convex valued, replication invariant and strongly monotone rules is described by some threshold function $g$ which assigns to every number of agents $n$ a minimal number of agents $g(n)$ needed to form a decisive coalition. Formally, the super majority rule $\varphi_{g}$ is defined for every profile $p$ in $\mathbb{L}^{N}$ by:

$$
\varphi_{g}=\mathbb{L}_{g(\# N)-\text { majority-relation }(p)}
$$

Here $g$ is a so-called threshold function from $\mathbb{N}-\{0\}$ to $\mathbb{N}-\{0\}$ defined by the following two conditions:

a) $\frac{m-1}{m}<\frac{g(n)}{n} \leq 1 \quad$ for all $n$ in $\mathbb{N}$,

b) $\frac{g(n)-1}{n}<\frac{g(k n)}{k n} \leq \frac{g(n)}{n} \quad$ for all $k$ and $n$ in $\mathbb{N}$. 
Clearly $\varphi_{g}$ is well-defined as by condition $\left.a\right), g(\# N) \leq \# N$ and further $\frac{m-1}{m} \cdot \# N<g(\# N)$ implies that $\mathbb{L}_{g(\# N)-\text { majority-relation( } p)}$ is non-empty. See also Example 4 and Proposition 4 . By condition $b$ ), minimality of the threshold is carried to different numbers of agents. So, $\varphi_{g}$ assigns to a profile $p$ in $\mathbb{L}^{N}$, the linear extensions of all pairs of alternatives on which at least $g(\# N)$ agents in $N$ unanimously agree. The fraction $\frac{g(n)}{n}$ can be seen as a minimal fraction for a coalition to be decisive. That is $\frac{g(k)-1}{k}<\frac{g(l)}{l}$ for all numbers $k$ and $l$ in $\mathbb{N}$. Indeed by condition $\left.b\right)$ on $g$ it follows that $\frac{g(k)-1}{k}<\frac{g(k l)}{k l} \leq \frac{g(k)}{k}$ and $\frac{g(l)-1}{l}<\frac{g(k l)}{k l} \leq \frac{g(l)}{l}$ for all such numbers $k$ and $l$. Hence, $\frac{g(k)-1}{k}<\frac{g(k l)}{k l} \leq \frac{g(l)}{l}$ and therewith $\frac{g(k)-1}{k}<\frac{g(l)}{l}$ for all numbers $k$ and $l$. It is therefore tempting to define $\varphi_{g}$ on the basis of $\beta=\inf \left\{\frac{g(n)}{n}: n \in \mathbb{N}\right\}$, and assigning to a profile $p$ in $\mathbb{L}^{N}$ the convex set $\mathbb{L}_{k-\text { majority-relation( }(p)}$ for $k$ such that $\frac{k}{\# N} \geq \beta$. But also assigning $\mathbb{L}_{l-\text { majority-relation }(p)}$ for $l$ such that $\frac{l}{\# N}>\beta$ would yield a rule satisfying the five conditions mentioned above. In case $\beta$ is rational these two definitions yield (slightly) different rules. Therefore to capture them both in one formulation we chose for the description based on a function like $g$.

Clearly, by definition, $\varphi_{g}$ is Pareto optimal and neutral. As $\mathbb{L}_{\cap\{p(i): i \in S\}}$ is convex by Lemma 1 and the fact that intersection of convex sets is convex, it follows that $\varphi_{g}$ is convex valued. Next, we argue that $\varphi_{g}$ is replication invariant.

Lemma $1 \varphi_{g}$ is replication invariant.

Proof. Consider profile $p$ in $\mathbb{L}^{N}$ and its $l^{\text {th }}$ replication $q=\left(p^{1}, p^{2}, \ldots, p^{l}\right)$ in $\mathbb{L}^{N_{1} \cup N_{2} \cup \ldots \cup N_{l}}$ for some integer $l \geq 2$. It is sufficient to prove that $\varphi_{g}(p)=\varphi_{g}(q)$. That is $g(\# N)$-majority-relation $(p)=$ $g(l \cdot \# N)$-majority-relation $(q)$. Since $\frac{g(l \cdot \# N)}{l \cdot \# N} \leq \frac{g(\# N)}{\# N}$, it follows that $g(\# N)$-majority-relation $(p) \subseteq$ $g(l \cdot \# N)$-majority-relation $(q)$. Indeed if $S=\{i \in N: . a . b .=p(i)\}$ for different alternatives $a$ and $b$ such that $\# S \geq g(\# N)$ and therewith $(a, b) \in g(\# N)$-majority-relation $(p)$, then we may identify $S_{1}$ up to $S_{l}$ in respectively $N_{1}$ up to $N_{l}$ such that $\# S=\# S_{j}$ and $. a . b .=q(i)$ for all $j \in\{1, \ldots, l\}$ and all $i \in S_{j}$. Therefore $\#\left\{i \in N_{1} \cup N_{2} \cup \ldots \cup N_{l}:\right.$ a.b. $\left.=q(i)\right\} \geq l \cdot \# S=l \cdot g(\# N)$ and $(a, b) \in g(l \cdot \# N)$-majority-relation $(q)$. Further, because of $\frac{g(\# N)-1}{\# N}<\frac{g(l \cdot \# N)}{l \cdot \# N}, g(l \cdot \# N)$-majorityrelation $(q) \subseteq g(\# N)$-majority-relation $(p)$. To see this let $\widetilde{S}=\left\{i \in N_{1} \cup N_{2} \cup \ldots \cup N_{l}:\right.$ a.b. $\left.=q(i)\right\}$ and $\# \widetilde{S} \geq l \cdot g(\# N)$. Because $q$ is the $l^{t h}$ replica of $p, \# S_{j}=\frac{1}{l} \cdot \# \widetilde{S}$, for $S_{j}=\widetilde{S} \cap N_{j}$ and $j \in$ $\{1,2, \ldots, l\}$. So, $\frac{\# S_{j}}{\# N} \geq \frac{g(l \cdot \# N)}{l \cdot \# N}>\frac{g(\# N)-1}{\# N}$ and therewith $\# S_{j}>g(\# N)-1$. Hence, $\# S_{j} \geq g(\# N)$ which implies $(a, b) \in g(\# N)$-majority-relation $(p)$.

Lemma $2 \varphi_{g}$ is strongly monotone.

Proof. Consider profile $p$ in $\mathbb{L}^{N}$ and any $R$ in $\varphi_{g}(p)$. Consider any update $q$ of $p$ towards $R$ such that for some agent $j$ in $N$ we have $p(i)=q(i)$ for all $i$ in $N-\{j\}$ and, $q(j)$ is an elementary change of $p(j)$ in pair $a b$ with $. a . b .=R, . a b .=q(j)$ and $. b a .=p(j)$. In view of Proposition 2 it is sufficient to prove that $R \in \varphi_{g}(q)$ and $\varphi_{g}(q) \subseteq \varphi_{g}(p)$. Now let $S \subseteq N$ with $\# S \geq g(\# N)$. Because of $R \in \varphi_{g}(p)$ it follows that $\cap\{p(i): i \in S\} \subseteq R$ and as .a.b. $=R$ we have $(b, a) \notin \cap\{p(i): i \in S\}$. So, by the choice of $q$

$$
\begin{aligned}
& \cap\{p(i): i \in S\} \subseteq \cap\{q(i): i \in S\} \text { and } \\
& \cap\{q(i): i \in S\} \subseteq(\cap\{p(i): i \in S\} \cup\{(a, b)\}) .
\end{aligned}
$$

As .a.b. $=R$ it follows that $\cap\{q(i): i \in S\} \subseteq R$. Because this holds for arbitrary $S$, such that $\# S \geq g(\# N)$, we have that $R \in \varphi_{g}(q)$. To prove $\varphi_{g}(q) \subseteq \varphi_{g}(p)$ let $\bar{R} \in \varphi_{g}(q)$. It is sufficient to 
prove $\bar{R} \in \varphi_{g}(p)$. As $\cap\{p(i): i \in S\} \subseteq \cap\{q(i): i \in S\}$ for all $S \subseteq N$, with $\# S \geq g(\# N)$, we have by definition of $\varphi_{g}$ that $\bar{R} \in \varphi_{g}(p)$.

All of the above makes it clear that the rule $\varphi_{g}$ is Pareto optimal, neutral, convex valued, replication invariant and strongly monotone. Next, we prove that only super majority rules satisfy these five conditions simultaneously. In the following two lemmas we provide some insight about coalitional power at profiles composed of two preferences which form an elementary change. Actually these profiles resemble collective decision situations between two alternatives, the ones on which the elementary change is based. In the following Lemmas we shall prove that on these profiles Pareto optimal, neutral, replication invariant, convex valued and strongly monotone rules $\varphi$ are equal to a super majority rule $\varphi_{g}$ for some function $g$. Thereafter, we show in Theorem 1 that this result expands to all profiles hence that $\varphi=\varphi_{g}$. Lemma 3 and 5 are based on the weak version of monotonicity and are also used in the following Section 4. Therefore, for now let rule $\varphi$ be Pareto optimal, neutral, replication invariant, convex valued and monotone.

Lemma 3 Let $\bar{R}$ be an elementary change of $R$ in pair ab. Let $S \subseteq N_{1}$ and $T \subseteq N_{2}$ be coalitions such that $\frac{1}{2} \leq \frac{\# S}{\# N_{1}} \leq \frac{\# T}{\# N_{2}}$. Then:

(a) $R \in \varphi\left(R^{S}, \bar{R}^{N_{1}-S}\right)$ ( $S$ is decisive; fraction $\frac{\# S}{\# N_{1}}$ is decisive);

(b) $\varphi\left(R^{S}, \bar{R}^{N_{1}-S}\right)=\{R\}$ implies $\varphi\left(R^{T}, \bar{R}^{N_{2}-T}\right)=\{R\}$ (if fraction $\frac{\# S}{\# N_{1}}$ is strictly decisive, then any greater fraction $\frac{\# T}{\# N_{2}}$ is also strictly decisive)

(c) $\varphi\left(R^{T}, \bar{R}^{N_{2}-T}\right)=\{R, \bar{R}\}$ implies $\varphi\left(R^{S}, \bar{R}^{N_{1}-S}\right)=\{R, \bar{R}\}$ (if fraction $\frac{\# T}{\# N_{2}}$ is not strictly decisive, then any smaller fraction $\frac{\# S}{\# N_{1}}$ larger than or equal to a half is not strictly decisive).

Proof. (a) Let $2 \cdot \# S \geq \# N_{1}$. Let $U$ be a coalition in $N_{1}$ such that $N_{1}-S \subseteq U$ and $\# S=\# U$. Pareto optimality implies $\varphi\left(R^{S}, \bar{R}^{N_{1}-S}\right) \in\{\{R\},\{\bar{R}\},\{R, \bar{R}\}\}$. Suppose $\bar{R} \in \varphi\left(R^{S}, \bar{R}^{N_{1}-S}\right)$. It is sufficient to prove that $R \in \varphi\left(R^{S}, \bar{R}^{N_{1}-S}\right)$. Considering the permutation $\tau$ on $A$ such that $\tau(x)=x$ for all $x \in A-\{a, b\}, \tau(a)=b$ and $\tau(b)=a$ it follows that $\tau R=\bar{R}$ and $\tau \bar{R}=R$. Now neutrality and $\bar{R} \in \varphi\left(R^{S}, \bar{R}^{N_{1}-S}\right)$ implies $R=\tau \bar{R} \in \varphi\left(\tau R^{S}, \tau \bar{R}^{N_{1}-S}\right)=\varphi\left(\bar{R}^{S}, R^{N_{1}-S}\right)$. So, monotonicity implies $R \in \varphi\left(\bar{R}^{N_{1}-U}, R^{U}\right)$. Now, anonymity which is implied by replication invariance, see Remark 1 , yields $R \in \varphi\left(R^{S}, \bar{R}^{N_{1}-S}\right)$.

(b) Let $\varphi\left(R^{S}, \bar{R}^{N_{1}-S}\right)=\{R\}$. Consider $N_{3}$ and $V$ and $W$ two subsets of $N_{3}$ such that $\# N_{3}=\# N_{1} \cdot \# N_{2}, \# V=\# N_{2} \cdot \# S$ and $\# W=\# N_{1} \cdot \# T$. Replication invariance now implies $\varphi\left(R^{V}, \bar{R}^{N_{3}-V}\right)=\{R\}$ and as $\# N_{2} \cdot \# S \leq \# N_{1} \cdot \# T$ monotonicity requires $R \in \varphi\left(R^{W}, \bar{R}^{N_{3}-W}\right)$ and as $\bar{R} \notin \varphi\left(R^{V}, \bar{R}^{N_{3}-V}\right)$ monotonicity and anonymity imply $\bar{R} \notin \varphi\left(R^{W}, \bar{R}^{N_{3}-W}\right)$. So, $\varphi\left(R^{W}, \bar{R}^{N_{3}-W}\right)=\{R\}$ and replication invariance implies $\varphi\left(R^{T}, \bar{R}^{N_{2}-T}\right)=\{R\}$.

(c) Let $\varphi\left(R^{T}, \bar{R}^{N_{2}-T}\right)=\{R, \bar{R}\}$. Then part (b) implies $\varphi\left(R^{S}, \bar{R}^{N_{1}-S}\right) \neq\{R\}$. Hence Pareto optimality yields $\varphi\left(R^{S}, \bar{R}^{N_{1}-S}\right)=\{\bar{R}\}$ or $\varphi\left(R^{S}, \bar{R}^{N_{1}-S}\right)=\{R, \bar{R}\}$. Because of part (a) the latter part of this disjunction holds.

Remark 3 Note that in the previous Lemma 3 (part(a)) only anonymity, neutrality and monotonicity is needed. This means that under these conditions $\varphi\left(R^{S}, \bar{R}^{N_{1}-S}\right)=\{R\}$ implies $\# S>\frac{n}{2}$. $\triangle$ 
The following Lemma generalizes the decision power of minority coalitions as discussed at the beginning of this section.

Lemma 4 Let $\bar{R}$ be an elementary change of $R$ in pair ab. Let $S \subseteq N$ be a coalition such that $\frac{1}{2} \leq \frac{\# S}{\# N} \leq \frac{m-1}{m}$. Then $\varphi\left(R^{S}, \bar{R}^{N-S}\right)=\{R, \bar{R}\}$.

Proof. First consider the special case where $\# N=m$ and $\# S=m-1$. So, $\frac{\# S}{\# N}=\frac{m-1}{m}$. Consider a numbering of the alternatives such that:

$$
\begin{aligned}
a_{1} a_{2} a_{3} a_{4} \ldots a_{t} a_{t+1} \ldots a_{m}= & \widetilde{R}^{1}=R \\
a_{2} a_{3} a_{4} \ldots a_{t} a_{t+1} \ldots a_{m} a_{1}= & \widetilde{R}^{2} \\
a_{3} a_{4} \ldots a_{t} a_{t+1} \ldots a_{m} a_{1} a_{2}= & \widetilde{R}^{3} \\
& \ldots \\
a_{m} a_{1} a_{2} \ldots a_{t} a_{t+1} \ldots a_{m-1}= & \widetilde{R}^{m} .
\end{aligned}
$$

Without loss of generality we may assume that $a=a_{t}, b=a_{t+1}$ and then $a_{1} a_{2} \ldots a_{t-1} a_{t+1} a_{t} \ldots a_{m}=$ $\bar{R}$. Let $p$ be the Condorcet profile defined for all agents $i$ in $N$ by $p(i)=\widetilde{R}^{i}$. Next we prove $\varphi(p)=\mathbb{L}$.

Let $\tau$ be a permutation on $A$ such that $\tau\left(a_{t}\right)=a_{t+1 \bmod m}$ for all $t$ in $\{1, \ldots, m\}$. Then $\tau \widetilde{R}^{i}=$ $\widetilde{R}^{i+1 \bmod m}$. Neutrality and replication invariance now imply that if $\widehat{R} \in \varphi(p)$, then $\tau^{t} \widehat{R} \in \varphi(p)$ for all $t \in\{1, \ldots, m\}$. For a given $s$ there is a integer $u$ such that $a_{s} \ldots=\tau^{u} \widehat{R}$. So, $\cap\left\{\tau^{t} \widehat{R}: t \in\right.$ $\{1, \ldots, m\}\}=\emptyset$. Convex valuedness of $\varphi$ and Proposition 1 now imply that $\varphi(p)=\mathbb{L}$.

Now $R=\widetilde{R}^{1} \supseteq \widetilde{R}^{t} \cap \bar{R}$ for all $t \neq i+1$ and $\bar{R} \supseteq \widetilde{R}^{i+1} \cap \widetilde{R}^{1}$. As $\varphi(p)=\mathbb{L}$, monotonicity implies $\varphi\left(\left(R^{S}, \bar{R}^{N-S}\right) \supseteq\{R, \bar{R}\}\right.$. Pareto optimality now yields that $\varphi\left(\left(R^{S}, \bar{R}^{N-S}\right)=\{R, \bar{R}\}\right.$. Finally, for any $S, N$ such that $\frac{1}{2} \leq \frac{\# S}{\# N} \leq \frac{m-1}{m}$, by Lemma 3 , we have $\varphi\left(R^{S}, \bar{R}^{N-S}\right)=\{R, \bar{R}\}$.

Remark 4 Note that in the previous two Lemmas 3 and 4 only monotonicity, neutrality and anonymity is needed and not the strong version of monotonicity. This implies that $\varphi\left(R^{S}, \bar{R}^{N-S}\right)=$ $\{R, \bar{R}\}$ for all preferences $R$ and $\bar{R}$ which form an elementary change and all $S$ such that $\frac{1}{m} \cdot \# N \leq \# S \leq \frac{m-1}{m} \cdot \# N$ and for all rules $\varphi$ that are Pareto optimal, neutral, anonymous, convex valued rules and monotone. As it is easy to check that many well-known rules satisfy the former four and do not satisfy this result on elementary changes, we may conclude that many well-known rules are not monotone. For instance, any score rule is not monotone. Indeed, an elementary change involving different scores implies that the absolute majorities, instead of $\frac{m-1}{m}$ majorities, are decisive at such profiles.

Assume now, in addition, that $\varphi$ is strongly monotone. In the sequel, we prove that $\varphi$ is in fact a super majority rule. For such a rule $\varphi$, we define two logically different sets $\mathcal{B}_{\text {all }}$ and $\mathcal{B}_{\text {some }}$. In the latter we find those pairs of numbers $(k, n)$ such that for some set of agents $N$, with $\# N=n$ and some coalition $S \subseteq N$, with $\# S=k$, and some pair of linear orders $R$ and $\bar{R}$ forming a elementary change, such that both coalitions $S$ and $N-S$ are decisive at profile $\left(R^{S}, \bar{R}^{N-S}\right)$, that is $\varphi\left(R^{S}, \bar{R}^{N-S}\right)=\{R, \bar{R}\}$. Hence the outcome at those profiles equals the Pareto set of the profile. In $\mathcal{B}_{\text {all }}$ we find all pairs of numbers $(k, n)$ for which this holds for all appropriate $S, N, R$ and $\bar{R}$. 


$$
\begin{aligned}
& \mathcal{B}_{\text {all }}=\left\{\begin{array}{c}
(k, n): \text { where } n-k \leq k \leq n-1 \text { and } \varphi\left(R^{S}, \bar{R}^{N-S}\right)=\{R, \bar{R}\} \\
\text { for all } R, \bar{R} \text { forming an elementary change } \\
\text { and all } S \subseteq N \text { such that } \# S=k \text { and } \# N=n\}
\end{array}\right\}
\end{aligned}
$$

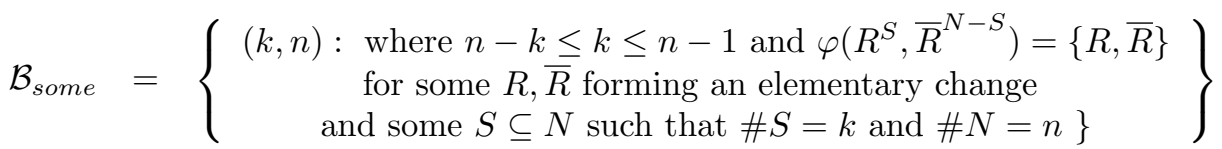

The two foregoing Lemmas imply that $\left\{(k, n): \frac{1}{2} \leq \frac{k}{n} \leq \frac{m-1}{m}\right\} \subseteq \mathcal{B}_{\text {all }}$ and logically we have $\mathcal{B}_{\text {all }}$ $\subseteq \mathcal{B}_{\text {some }}$. We shall prove that the latter subset relation is in fact an equation. Thereafter we refer to these sets by $\mathcal{B}$.

The following Lemma shows that for all pairs of sets of agents $(S, N)$ such that $S \subset N$ and $(\# S, \# N) \in \mathcal{B}_{\text {all }}$ and all linear orders $R$ and $\bar{R}$, not necessarily forming an elementary change, rule $\varphi$ assigns the Pareto set to profile $\left(R^{S}, \bar{R}^{N-S}\right)$.

Lemma 5 Let $S \subseteq N$ be such that $(\# S, \# N) \in \mathcal{B}_{\text {all }}$. Let $R$ and $\bar{R}$ be two linear orders. Then $\varphi\left(R^{S}, \bar{R}^{N-S}\right)=\mathbb{L}_{R \cap \bar{R}}$.

Proof. By induction on $\delta(R, \bar{R})$.

Basis If $\delta(R, \bar{R})=0$, then it follows by Pareto optimality. Let $\delta(R, \bar{R})=1$. As $(\# S, \# N) \in \mathcal{B}_{\text {all }}$ it follows by definition that $\varphi\left(R^{S}, \bar{R}^{N-S}\right)=\{R, \bar{R}\}=\mathbb{L}_{R \cap \bar{R}}$.

Induction Step Let $\delta(R, \bar{R})=t \geq 2$. Pareto optimality implies that $\varphi\left(R^{S}, \bar{R}^{N-S}\right) \subseteq \mathbb{L}_{R \cap \bar{R}}$. Let $\widetilde{R} \in \mathbb{L}_{R \cap \bar{R}}-\{R, \bar{R}\}$. It follows that $\delta(R, \widetilde{R})<t$ and $\delta(\bar{R}, \widetilde{R})<t$. So, by induction $\mathbb{L}_{R \cap \widetilde{R}}=$ $\varphi\left(R^{S}, \widetilde{R}^{N-S}\right)$ and $\mathbb{L}_{\bar{R} \cap \widetilde{R}}=\varphi\left(\widetilde{R}^{S}, \bar{R}^{N-S}\right)$. If $R \in \varphi\left(R^{S}, \bar{R}^{N-S}\right)$, then by strong monotonicity $\widetilde{R} \in \mathbb{L}_{R \cap \widetilde{R}}=\varphi\left(R^{S}, \widetilde{R}^{N-S}\right) \subseteq \varphi\left(R^{S}, \bar{R}^{N-S}\right)$. If $\bar{R} \in \varphi\left(R^{S}, \bar{R}^{N-S}\right)$, then by strong monotonicity $\widetilde{R} \in \mathbb{L}_{\bar{R} \cap \widetilde{R}}=\varphi\left(\widetilde{R}^{S}, \bar{R}^{N-S}\right) \subseteq \varphi\left(R^{S}, \bar{R}^{N-S}\right)$. If $\widetilde{R} \in \varphi\left(R^{S}, \bar{R}^{N-S}\right)$, then by strong monotonicity $R \in \mathbb{L}_{R \cap \widetilde{R}}=\varphi\left(R^{S}, \widetilde{R}^{N-S}\right) \subseteq \varphi\left(R^{S}, \bar{R}^{N-S}\right)$ and $\bar{R} \in \mathbb{L}_{\bar{R} \cap \widetilde{R}}=\varphi\left(\widetilde{R}^{S}, \bar{R}^{N-S}\right) \subseteq \varphi\left(R^{S}, \bar{R}^{N-S}\right)$.This then yields by convexity that $\mathbb{L}_{R \cap \bar{R}} \subseteq \varphi\left(R^{S}, \bar{R}^{N-S}\right)$. Hence if $\widetilde{R} \in \varphi\left(R^{S}, \bar{R}^{N-S}\right)$, then $\mathbb{L}_{R \cap \bar{R}}=$ $\varphi\left(R^{S}, \bar{R}^{N-S}\right)$. Since $\bar{R} \in \varphi\left(R^{S}, \bar{R}^{N-S}\right), \widetilde{R} \in \varphi\left(R^{S}, \bar{R}^{N-S}\right)$, or $\widetilde{R} \in \varphi\left(R^{S}, \bar{R}^{N-S}\right)$ for some $\widetilde{R} \in$ $\mathbb{L}_{R \cap \bar{R}}-\{R, \bar{R}\}$, the above yields that $\varphi\left(R^{S}, \bar{R}^{N-S}\right)=\mathbb{L}_{R \cap \bar{R}}$.

Next lemma is about profiles polarized in elementary changes. We show that whenever some coalition of size $k$ is not powerful enough to impose its preference uniquely at some profile of elementary changes, then any coalition of the same size is also not powerful enough to impose its preference uniquely at any profile of elementary changes. Since $\mathcal{B}_{\text {all }} \subseteq \mathcal{B}_{\text {some }}$, this boils down to say that $\mathcal{B}_{\text {all }}=\mathcal{B}_{\text {some }}$.

Lemma 6 Let $S$ be a subset of $N$ such that $(\# S, \# N) \in \mathcal{B}_{\text {some. }}$ Then $(\# S, \# N) \in \mathcal{B}_{\text {all }}$.

Proof. Let $(\# S, \# N) \in \mathcal{B}_{\text {some }}$ such that $\# N-\# S \leq \# S \leq \# N-1$. Let $R$ be an elementary change of $\bar{R}$ in pair $a b$ at position $l$, such that $\varphi\left(R^{S}, \bar{R}^{N-S}\right)=\{R, \bar{R}\}$. It is sufficient to prove that $(\# S, \# N) \in \mathcal{B}_{\text {all }}$. If $\frac{\# S}{\# N} \leq \frac{m-1}{m}$, then this holds by Lemma 4 . Therefore we may assume that $\frac{\# S}{\# N}>\frac{m-1}{m}$. So, there are $T \subseteq S$ be such that $\# N-\# T \leq \# T \leq \frac{m-1}{m} \cdot \# N$ and by Lemma 4 
$(\# T, \# N) \in \mathcal{B}_{\text {all }}$. Note that, by neutrality, $\varphi\left(R_{l}^{S}, \bar{R}_{l}^{N-S}\right)=\left\{R_{l}, \bar{R}_{l}\right\}$ for all elementary changes in position $l$. First we will show that this also extends to all elementary changes in position $l+1$.

Let $. a b c .=R$ and $. b a c .=\bar{R}$. Consider $. a b c .=R=R_{1}, . a c b .=R_{2}, . c a b .=R_{3}, . c b a .=R_{4}$, $. b c a .=R_{5}$ and $. b a c .=R_{6}=\bar{R}$. It is sufficient to prove that $\varphi\left(R_{1}^{S}, R_{2}^{N-S}\right)=\left\{R_{1}, R_{2}\right\}$ since $R_{1}$ and $R_{2}$ form an elementary change in position $l+1$.

Since $(\# T, \# N) \in \mathcal{B}_{\text {all }}$, by Lemma $5, \varphi\left(R_{5}^{N-T}, R_{2}^{T}\right)=\left\{R_{1}, R_{2}, \ldots, R_{6}\right\}$. From this, monotonicity implies that $\left\{R_{1}, R_{2}\right\} \subseteq \varphi\left(R_{6}^{N-S}, R_{1}^{S-T}, R_{2}^{T}\right)$. Since $R_{1} \in \varphi\left(R_{6}^{N-S}, R_{1}^{S-T}, R_{2}^{T}\right)$, by strong monotonicity we have that $\varphi\left(R_{6}^{N-S}, R_{1}^{S}\right) \subseteq \varphi\left(R_{6}^{N-S}, R_{1}^{S-T}, R_{2}^{T}\right)$. By assumption $\varphi\left(R_{6}^{N-S}, R_{1}^{S}\right)=\left\{R_{6}, R_{1}\right\}$, and therewith $\left\{R_{1}, R_{2}, R_{6}\right\} \subseteq \varphi\left(R_{6}^{N-S}, R_{1}^{S-T}, R_{2}^{T}\right)$. So, by Pareto optimality $\left\{R_{1}, R_{2}, R_{6}\right\}=\varphi\left(R_{6}^{N-S}, R_{1}^{S-T}, R_{2}^{T}\right)$. Since $(\# T, \# N) \in \mathcal{B}_{\text {all }}$, by Lemma 5 we have that $\varphi\left(R_{6}^{N-T}, R_{3}^{T}\right)=\left\{R_{1}, R_{2}, \ldots, R_{6}\right\}$. So, by monotonicity we have that $\left\{R_{1}, R_{2}, R_{3}\right\} \subseteq \varphi\left(R_{6}^{N-S}, R_{1}^{S-T}, R_{3}^{T}\right)$. As $R_{2} \in \varphi\left(R_{6}^{N-S}, R_{1}^{S-T}, R_{3}^{T}\right)$, strong monotonicity implies $\varphi\left(R_{6}^{N-\bar{S}}, R_{1}^{S-T}, R_{3}^{T}\right) \supseteq \varphi\left(R_{6}^{N-S}, R_{1}^{S-T}, R_{2}^{T}\right)=\left\{R_{1}, R_{2}, R_{6}\right\}$. Hence, we have that $\left\{R_{1}, R_{2}, R_{3}, R_{6}\right\} \subseteq \varphi\left(R_{6}^{N-S}, R_{1}^{S-T}, R_{3}^{T}\right)$ and convex valuedness now implies $\varphi\left(R_{6}^{N-S}, R_{1}^{S-T}, R_{3}^{T}\right)=$ $\left\{R_{1}, R_{2}, \ldots, R_{6}\right\}$. By employing the permutation $\tau$ on $A$ such that $\tau(x)=x$ for all $x \in A-\{a, b, c\}$, $\tau(a)=b, \tau(b)=c$ and $\tau(c)=a$ neutrality yields $\varphi\left(R_{4}^{N-S}, R_{5}^{S-T}, R_{1}^{T}\right)=\left\{R_{1}, R_{2}, \ldots, R_{6}\right\}$. Monotonicity and Pareto optimality finally yields that $\varphi\left(R_{1}^{S}, R_{2}^{N-S}\right)=\left\{R_{1}, R_{2}\right\}$. Then, by neutrality, for all elementary changes $\left(R_{l+1}^{S}, \bar{R}_{l+1}^{N-S}\right)$ in position $l+1$, we have that $\varphi\left(R_{l+1}^{S}, \bar{R}_{l+1}^{N-S}\right)=\left\{R_{l+1}, \bar{R}_{l+1}\right\}$.

By a similar approach it can be shown that for elementary changes $R^{\prime}$ and $\bar{R}^{\prime}$ in position $l-1$ we have $\varphi\left(R^{\prime S}, \bar{R}^{\prime N-S}\right)=\left\{R^{\prime}, \bar{R}^{\prime}\right\}$. Therefore we may conclude that $(\# S, \# N) \in \mathcal{B}_{\text {all }}$.

Remark 5 Now let $\mathcal{B}=\mathcal{B}_{\text {all }}=\mathcal{B}_{\text {some. }}$ Consider the function $f$ from $\mathbb{N}-\{0\}$ to $\mathbb{N}-\{0\}$ for an arbitrary number $n$ by $f(n)=\min \{\# S: S \subseteq N$, with $\# N=n$, and $(\# S, \# N) \notin \mathcal{B}\}$. By definition and as $\left\{(k, n): \frac{1}{2} \leq \frac{k}{n} \leq \frac{m-1}{m}\right\} \subseteq \mathcal{B}_{\text {all }}=\mathcal{B}$, it follows that $\frac{m-1}{m}<\frac{f(n)}{n} \leq 1$ for all $n$ in $\mathbb{N}$ (first condition of the threshold function). By definition of $f$ and since $\varphi$ is replication invariant it follows that $k \cdot(f(n)-1)<f(k n) \leq k \cdot f(n)$. Therefore it follows that $\frac{f(n)-1}{n}<\frac{f(k n)}{k n} \leq \frac{f(n)}{n}$ (second condition of the threshold function). Hence $f$ is indeed a threshold function.

The following Theorem shows that $\varphi$ equals super majority rule $\varphi_{g}$.

Theorem 1 A rule $\varphi$ is Pareto optimal, neutral, replication invariant, convex valued and strongly monotone if and only if $\varphi=\varphi_{g}$ for some threshold function $g$.

Proof. (If part) Follows from the definition of $\varphi_{g}$ and Lemmas 1 and 2.

(Only if-part) Let $\varphi$ be Pareto optimal, neutral, replication invariant, convex valued and strongly monotone. By Lemmas above and Remark 5, we may find such a threshold function $g$ such that for all elementary changes $R$ and $\bar{R}$ and all coalitions $T \subseteq N$ we have that $\varphi_{g}\left(R^{T}, \bar{R}^{N-T}\right)=$ $\varphi\left(R^{T}, \bar{R}^{N-T}\right)$. Let $p$ be any profile in $\mathbb{L}^{N}$, it remains to prove that $\varphi(p)=\varphi_{g}(p)$.

To show $\varphi(p) \subseteq \varphi_{g}(p)$, let $S \subseteq N$ be any coalition such that $\# S \geq g(\# N)$ and $. a . b .=p(i)$ for some alternatives $a$ and $b$ and for all $i$ in $S$. It is sufficient to prove that $\varphi(p) \subseteq \mathbb{L}_{a b}$. To the contrary let $\widehat{R} \in \varphi(p)-\mathbb{L}_{a b} \subseteq \mathbb{L}_{b a}$. We distinguish two cases.

Case $(a$ and $b$ are consecutive in $\widehat{R})$ : Let $. b a .=\widehat{R}$. Then order $R^{\prime}$ in $\mathbb{L}_{a b}$ being the elementary change of $\widehat{R}$ in $b a$, is between $\widehat{R}$ and $p(i)$ for all $i \in S$. Hence, monotonicity would yield that $\widehat{R} \in$ $\varphi\left(\left(R^{\prime}\right)^{S}, \widehat{R}^{N-S}\right)$. So, $\varphi\left(\left(R^{\prime}\right)^{S}, \widehat{R}^{N-S}\right) \neq \varphi_{g}\left(\left(R^{\prime}\right)^{S}, \widehat{R}^{N-S}\right)=\left\{R^{\prime}\right\}$ which cannot be as $R^{\prime}$ and $\widehat{R}$ form an elementary change in $b a$. 
Case $(a$ and $b$ are not consecutive in $\widehat{R})$ : Let $c_{1}, c_{2} \ldots c_{l}$ be alternatives different from $a$ and $b$ such that $. b c_{1} c_{2} \ldots c_{l} a .=\widehat{R}$. We may take $\widehat{R}$ such that $l$ is minimal. For $t \in\{1,2, \ldots, l\}$ let $\widehat{R}^{t}$ be the linear order defined by $\left.\widehat{R}^{t}\right|_{A-\left\{c_{t}\right\}}=\left.\widehat{R}\right|_{A-\left\{c_{t}\right\}}$ and $c_{t} b . a .=\widehat{R}^{t}$. Furthermore, let $\widehat{R}^{0}$ be the linear order defined by $\left.\widehat{R}^{0}\right|_{A-\left\{c_{l}\right\}}=\left.\widehat{R}\right|_{A-\left\{c_{l}\right\}}$ and $. b c_{1} c_{2} \ldots c_{l-1} a c_{l} .=\widehat{R}^{0}$.Take any $i \in S$ and hence $p(i)$ in $\mathbb{L}_{a b}$. We prove that for each $p(i)$, there is a $t \in\{0,1,2, \ldots, l\}$ such that $\widehat{R}^{t}$ is between $p(i)$ and $\widehat{R}$. Take $s$ such that $\left(c_{s}, c_{u}\right) \in p(i)$ for all $u \in\{1,2, \ldots, l\}$. Note that we either have $. c_{s} . a .=p(i)$ or .a.c $c_{s} .=p(i)$. Assume $. c_{s} \cdot a .=p(i)$. Hence, $. c_{s} \cdot a \cdot b .=p(i)$. To prove that $\widehat{R}^{s}$ is between $p(i)$ and $\widehat{R}$ let $(x, y) \in \widehat{R}^{s}-\widehat{R}$. It is sufficient to show that $(x, y) \in p(i)$. By construction of $\widehat{R}^{s}$ and $\widehat{R}$, we have that $x=c_{s}$ and either $y=b$ or $y=c_{u}$ for some $0<u<s$. Because of $. c_{s} \cdot a . b .=p(i)$ and $\left(c_{s}, c_{u}\right) \in p(i)$ for all $u \in\{1,2, \ldots, l\}$, and by the choice of $s$, we have that $(x, y) \in p(i)$. Hence for the case $. c_{s} \cdot a .=p(i)$, we have that $\widehat{R}^{t}$ is between $p(i)$ and $\widehat{R}$ for $t=s$. Now assume $. a . c_{s} .=p(i)$. So, by construction .a.c..$=p(i)$ for all $t \in\{1,2, \ldots, l\}$. Then $\widehat{R}^{0} \subseteq \widehat{R} \cup\left\{\left(a, c_{l}\right)\right\} \subseteq \widehat{R} \cup p(i)$. Therefore, $\widehat{R}^{0}$ is between $\widehat{R}$ and $p(i)$. Hence for the case .a.c. $c_{s}=p(i), \widehat{R}^{t}$ is between $p(i)$ and $\widehat{R}$ for $t=0$. Therefore we may take $s_{i} \in\{0,1, \ldots, l\}$ such that $\widehat{R}^{s_{i}}$ is between $p(i)$ and $\widehat{R}$ for all $i \in S$.

Next take $S_{t}=\left\{i \in S: t=s_{i}\right\}$, i.e., the set of individuals $i \in S$ which share $\widehat{R}^{t}$ as the preference between $\widehat{R}$ and their preference $p(i)$. Let $S_{v}$ be such that $\# S_{v} \geq \# S_{t}$ for all $t \in\{0,1, \ldots, l\}$. By the assumption on the minimality of $l$, we have that $\widehat{R}^{v} \notin \varphi(p)$ and as $\widehat{R}^{v}$ is between $\widehat{R}$ and $p(i)$ for all $i \in S_{v}$ it follows by strong monotonicity that $\widehat{R}^{v} \notin \varphi\left(\left(\widehat{R}^{v}\right)^{S_{v}}, \widehat{R}^{N-S_{v}}\right)$. But by construction of $S_{v}$ we have that $\# S_{v} \geq \frac{1}{l+1} \cdot \frac{m-1}{m} \cdot \# N \geq \frac{1}{m-1} \cdot \frac{m-1}{m} \cdot \# N \geq \frac{\# N}{m}$. Therefore, $\#\left(N-S_{v}\right) \leq \frac{m-1}{m} \cdot \# N$. So, as $\left\{(k, n): \frac{1}{2} \leq \frac{k}{n} \leq \frac{m-1}{m}\right\} \subseteq \mathcal{B}$ it follows that $\#\left(N-S_{v}\right)<g(\# N)$ and therewith that the contradiction $\widehat{R}^{v} \in \varphi\left(\left(\widehat{R}^{v}\right)^{S_{v}}, \widehat{R}^{N-S_{v}}\right)$.

To show $\varphi(p) \supseteq \varphi_{g}(p)$, let $R \in \varphi_{g}(p)$ and suppose to the contrary that $R \notin \varphi(p)$. As by the previous part $\varphi(p) \subseteq \varphi_{g}(p)$ and convex valuedness we may assume that there exist $\bar{R} \in \varphi(p) \subseteq \varphi_{g}(p)$ such that $R$ and $\bar{R}$ form an elementary change in $a b$. Furthermore, without loss of generality, let $. a b .=R$ and $. b a .=\bar{R}$ and let $S=\left\{i \in N: p(i) \in \mathbb{L}_{a b}\right\}$. Strong monotonicity now implies that $R, \bar{R} \in \varphi_{g}\left(R^{S}, \bar{R}^{N-S}\right) \subseteq \varphi_{g}(p)$ and $\bar{R} \in \varphi\left(R^{S}, \bar{R}^{N-S}\right) \subseteq \varphi(p)$ and $R \notin \varphi\left(R^{S}, \bar{R}^{N-S}\right)$. This however contradicts $\varphi_{g}\left(R^{T}, \bar{R}^{N-T}\right)=\varphi\left(R^{T}, \bar{R}^{N-T}\right)$ for all coalitions $T \subseteq N$. This contradiction completes the proof.

\section{Dropping Convex Valuedness}

Although super majority rules satisfy several nice conditions, they are not extremely resolute in the sense that they often assign a lot of outcomes to profiles due to lack of coalitional power. This is essentially caused by the lower bound $n \cdot \frac{m-1}{m}$ for $k$, a lower bound which is needed in order to avoid cyclical decisions. The discussion at the beginning of the previous section involving the Condorcet profile on three alternatives makes clear that an increase on the resolution of rules can most likely be achieved by dropping the convex valuedness condition. In general, we did not succeed in describing the class of all Pareto optimal, neutral, replication invariant and monotone rules. Therefore we fixed our attention to the well-known Kemeny rule belonging to this class. It can be seen as a natural application of the Kemeny distance, introduced by Kemeny and Snell (1962), to social choice theory. This rule assigns those ranking(s) to a profile which minimizes the sum of distances to each of the individual preference in that profile.

Definition 1 Given a profile $p \in \mathbb{L}^{N}$, a preference relation $R$ is a Kemeny ranking for $p$, if for all 
$R^{\prime} \in \mathbb{L}$, we have:

$$
\sum_{i \in N} \delta(R, p(i)) \leq \sum_{i \in N} \delta\left(R^{\prime}, p(i)\right)
$$

A rule which assigns all Kemeny rankings to each profile is called the Kemeny rule.

Definition 2 Kemeny rule, denoted by $\varphi_{\text {Kemeny }}$, assigns to a profile $p \in \mathbb{L}^{N}$ :

$$
\varphi_{\text {Kemeny }}(p)=\{R \in \mathbb{L}: R \text { is a Kemeny ranking for } p\} .
$$

We characterize the Kemeny rule by Pareto optimality, neutrality, pairwiseness, consistency and monotonicity. For that we first prove that the Kemeny rule satisfies these conditions.

Lemma $7 \varphi_{\text {Kemeny }}$ is Pareto optimal, neutral, pairwise, and consistent.

To show Pareto optimality, take any profile $p \in \mathbb{L}^{N}$ such that for some $a, b \in A$ and for all $i \in N, p(i)=. a . b$. . Consider the permutation $\sigma_{a b}$ on $A$ such that $\sigma_{a b}(a)=b, \sigma_{a b}(b)=a$, and $\sigma_{a b}(c)=c$ for all $c \in A-\{a, b\}$. Then by Proposition 5 in the appendix, for any $i \in N$ and for any linear order $R^{a b} \in \mathbb{L}_{a b}$ and $R^{b a}=\sigma_{a b} R^{a b}$, we have that:

$$
\delta\left(p(i), R^{a b}\right)<\delta\left(p(i), R^{b a}\right) .
$$

Hence,

$$
\sum_{i \in N} \delta\left(p(i), R^{a b}\right)<\sum_{i \in N} \delta\left(p(i), R^{b a}\right) .
$$

Therefore, $\varphi_{\text {Kemeny }}(p) \cap \mathbb{L}_{b a}=\emptyset$ which implies $\varphi_{\text {Kemeny }}(p) \subseteq \mathbb{L}_{a b}$ and therewith it is Pareto optimal. It satisfies neutrality by definition and pairwiseness because $M(p)=M(q)$ implies

$$
\begin{aligned}
\sum_{i \in N} \delta(R, p(i)) & =\sum_{(a, b) \in A \times A} \sum_{i \in N}(a, b) \in p(i)-R \\
& =\sum_{(a, b) \in A \times A-R} \sum_{i \in N}(a, b) \in p(i) \\
& =\sum_{(a, b) \in A \times A-R} M(p)_{(a, b)} \\
& =\sum_{(a, b) \in A \times A-R} M(q)_{(a, b)} \\
& =\sum_{i \in N} \delta(R, q(i)) .
\end{aligned}
$$

Hence, in that case $\sum_{i \in N} \delta(R, p(i))=\sum_{i \in N} \delta(R, q(i))$ for all profiles $p$ and $q$ and for all linear orders $R \in \mathbb{L}$. Consistency follows because for $R \in \mathbb{L}$ and profiles $p \in \mathbb{L}^{N_{1}}$ and $q \in \mathbb{L}^{N_{2}}$, where $N_{1}$ and $N_{2}$ are two disjoint finite and non-empty sets of agents,

$$
\delta(R,(p, q))=\delta(R, p)+\delta(R, q) .
$$

The following lemma shows that the Kemeny rule is (strongly) monotone. 
Lemma $8 \varphi_{\text {Kemeny }}$ is strongly monotone.

Proof. Consider profile $p$ in $\mathbb{L}^{N}$, and any $R \in \varphi_{\text {Kemeny }}(p)$. In the view of Proposition 2 we can consider any update $q$ of $p$ towards $R$ such that $p(i)=q(i)$ for all $i$ in $N-\{j\}$ and for some agent $j$ in $N, q(j)$ is an elementary change of $p(j)$ in pair $a b$ with $. a . b .=R, . a b .=q(j)$ and $. b a .=p(j)$. It is sufficient to prove that $R \in \varphi_{\text {Kemeny }}(q)$ and $\varphi_{\text {Kemeny }}(q) \subseteq \varphi_{\text {Kemeny }}(p)$. First we prove $R \in \varphi_{\text {Kemeny }}(q)$. To the contrary suppose there exist $R^{\prime} \in \mathbb{L}$ such that

$$
\sum_{i \in N} \delta(R, q(i))>\sum_{i \in N} \delta\left(R^{\prime}, q(i)\right)
$$

Hence,

$$
\sum_{i \in N} \delta(R, q(i)) \geq \sum_{i \in N} \delta\left(R^{\prime}, q(i)\right)+1
$$

Since $R$ is a Kemeny ranking for $p$, we have $R \in \varphi_{\text {Kemeny }}(p)$ therefore,

$$
\sum_{i \in N} \delta(R, p(i)) \leq \sum_{i \in N} \delta\left(R^{\prime}, p(i)\right) .
$$

But then we have

$$
\begin{aligned}
\delta\left(R^{\prime}, p(j)\right)+\sum_{i \in N-\{j\}} \delta\left(R^{\prime}, p(i)\right) & =\sum_{i \in N} \delta\left(R^{\prime}, p(i)\right) \\
& \geq \sum_{i \in N} \delta(R, p(i)) \\
& =\delta(R, p(j))+\sum_{i \in N-\{j\}} \delta(R, p(i)) \\
& =\delta(R, q(j))+1+\sum_{i \in N-\{j\}} \delta(R, q(i)) \\
& =\sum_{i \in N} \delta(R, q(i))+1 \\
& \geq \sum_{i \in N} \delta\left(R^{\prime}, q(i)\right)+2 \\
& =\delta\left(R^{\prime}, q(j)\right)+\sum_{i \in N-\{j\}} \delta\left(R^{\prime}, q(i)\right)+2 \\
& =\delta\left(R^{\prime}, q(j)\right)+\sum_{i \in N-\{j\}} \delta\left(R^{\prime}, p(i)\right)+2 .
\end{aligned}
$$

Hence,

$$
\begin{aligned}
\delta\left(R^{\prime}, p(j)\right) & \geq \delta\left(R^{\prime}, q(j)\right)+2 \\
& =\delta\left(R^{\prime}, q(j)\right)+\delta(q(j), p(j))+1,
\end{aligned}
$$

which, by the triangle inequality yields the contradiction

$$
\delta\left(R^{\prime}, p(j)\right) \geq \delta\left(R^{\prime}, p(j)\right)+1 .
$$


This completes the proof of $R \in \varphi_{\text {Kemeny }}(q)$. Next we show $\varphi_{\text {Kemeny }}(q) \subseteq \varphi_{\text {Kemeny }}(p)$. To the contrary, suppose there exist $R^{\prime} \in \varphi_{\text {Kemeny }}(q)-\varphi_{\text {Kemeny }}(p)$. Since $R \in \varphi_{\text {Kemeny }}(p)$ and $R^{\prime} \notin \varphi_{\text {Kemeny }}(p)$ we have

$$
\sum_{i \in N} \delta(R, p(i))+1 \leq \sum_{i \in N} \delta\left(R^{\prime}, p(i)\right) .
$$

Now $R \in \varphi_{\text {Kemeny }}(q)$ and $R^{\prime} \in \varphi_{\text {Kemeny }}(q)$ imply

$$
\sum_{i \in N} \delta(R, q(i))=\sum_{i \in N} \delta\left(R^{\prime}, q(i)\right)
$$

Hence,

$$
\delta(R, q(j))+\sum_{\substack{i \in N \\ i \neq j}} \delta(R, p(i))=\delta\left(R^{\prime}, q(j)\right)+\sum_{\substack{i \in N \\ i \neq j}} \delta\left(R^{\prime}, p(i)\right) .
$$

So,

$$
\delta(R, p(j))-1+\sum_{\substack{i \in N \\ i \neq j}} \delta(R, p(i))=\delta\left(R^{\prime}, q(j)\right)+\sum_{\substack{i \in N \\ i \neq j}} \delta\left(R^{\prime}, p(i)\right) .
$$

That is

$$
\sum_{i \in N} \delta(R, p(i))-1=\sum_{\substack{i \in N \\ i \neq j}} \delta\left(R^{\prime}, p(i)\right)+\delta\left(R^{\prime}, q(j)\right) .
$$

Using inequality (1) this yields

$$
\sum_{i \in N} \delta\left(R^{\prime}, p(i)\right)-2 \geq \sum_{\substack{i \in N \\ i \neq j}} \delta\left(R^{\prime}, p(i)\right)+\delta\left(R^{\prime}, q(j)\right) .
$$

Hence,

$$
\delta\left(R^{\prime}, p(j)\right)-2 \geq \delta\left(R^{\prime}, q(j)\right)
$$

So,

$$
\begin{aligned}
\delta\left(R^{\prime}, p(j)\right) & >\delta\left(R^{\prime}, q(j)\right)+1 \\
& =\delta\left(R^{\prime}, q(j)\right)+\delta(p(j), q(j)) \\
& \geq \delta\left(R^{\prime}, p(j)\right) .
\end{aligned}
$$

This contradiction ends the proof

In order to prove that Kemeny rule is the only rule satisfying these five conditions simultaneously, let $\varphi$ be a rule that is Pareto optimal, neutral, pairwise, consistent, and monotone. The following five Lemma's prepare the proof of this characterization.

First, we prove that equality holds between $\varphi$ and $\varphi_{\text {kemeny }}$ for maximal conflicts. These are profiles $p$ at which two equal groups of agents have totally opposing preferences over all pairs of alternatives. So, there are $2 k$ agents and a preference $R$ in $\mathbb{L}$ such that $k$ agents have preference $p(i)=R$ and the remaining $k$ agents have preference $p(i)=-R$, where $-R=\{(b, a):(a, b) \in R\}$.

Lemma 9 Let $p$ be a maximal conflict. Then $\varphi(p)=\mathbb{L}=\varphi_{\text {Kemeny }}(p)$. 
Proof. It is straightforward to show that $\varphi_{\text {Kemeny }}=\mathbb{L}$. For alternatives $a$ and $b$ consider the permutation $\sigma_{a b}$ on $A$ such that $\sigma_{a b}(a)=b, \sigma_{a b}(b)=a$ and $\sigma_{a b}(c)=c$ for all $c \in A-\{a, b\}$. Neutrality and pairwiseness imply that $\sigma_{a b} \circ \varphi(p)=\varphi\left(\sigma_{a b} p\right)=\varphi(p)$. This means that $\varphi(p)$ is closed under any permutation of $A$ which implies that $\varphi(p)=\mathbb{L}$.

Next, we prove that the equality of $\varphi$ and $\varphi_{\text {Kemeny }}$ extends to almost maximal conflicts. These are profiles $p$ at which equal groups of agents have totally opposing preferences over all but one pair of alternatives. So, there are $2 k$ agents, two alternatives $a$ and $b$ and a preference $R$ in $\mathbb{L}$ such that $a b \ldots=R$, meaning that $a$ is best and $b$ is second best at $R, k$ agents have preference $p(i)=R$ and the remaining $k$ agents have preference $p(i)=\sigma_{a b}(-R)$. Let us denote these profiles by $\pi(k, a b)$.

Lemma 10 Let $p$ be an almost maximal conflict, say $p=\pi(k, a b)$. Then $\varphi(p)=\mathbb{L}_{a b}=\varphi_{\text {Kemeny }}(p)$.

Proof. It is straightforward to show that $\mathbb{L}_{a b}=\varphi_{\text {Kemeny }}(p)$. Pareto optimality implies that $\varphi(p) \subseteq \mathbb{L}_{a b}$. In order to prove the reverse inclusion consider $R$ in $\mathbb{L}_{a b}$. Let $q$ be the maximal conflict on the same set of $2 k$ agents such that the agents at $p$, which have preference $R$, have preference $R$ at $q$ and those having preference $\sigma_{a b}(-R)$ at $p$ have preference $-R$ at $q$. Monotonicity and Lemma 9 imply that $R$ is in $\varphi(p)$. As $R$ is arbitrarily chosen in $\mathbb{L}_{a b}$, it follows that $\mathbb{L}_{a b} \subseteq \varphi(p)$. So, $\mathbb{L}_{a b}=\varphi(p)$.

In the following lemma we show that $\varphi$ and $\varphi_{\text {Kemeny }}$ are strongly pairwise, i.e., the outcome only depends on the net pairwise comparisons of alternatives. For an arbitrary profile $p$ let $D(p)$ denote the $m \times m$ difference matrix defined for every cell $(a, b)$ by

$$
D(p)_{a b}=\max \left\{0, M(p)_{a b}-M(p)_{b a}\right\} .
$$

It is easy to see that for any profile $p$ we have $\varphi_{\text {Kemeny }}(p)=\left\{R: \lambda(p, R) \leq \lambda\left(p, R^{\prime}\right)\right.$ for all $R^{\prime} \in$ $\mathbb{L}\}$ where $\lambda(p, R)=\sum_{a b \notin R} D(p)_{a b}$. Indeed for all linear orders $R \in \mathbb{L}, \delta(p, R)=\sum_{a b \notin R} M(p)_{a b}=$ $\sum_{a b \notin R} D(p)_{a b}+\min \left\{M(p)_{a b}, M(p)_{b a}\right\}=\sum_{a b \notin R} D(p)_{a b}+\sum_{a b \notin R} \min \left\{M(p)_{a b}, M(p)_{b a}\right\}$, where the former term equals $\lambda(p, R)$ and the latter term is constant.

Consider two profiles $p$ in $\mathbb{L}^{N}$ and $p^{\prime}$ in $\mathbb{L}^{N^{\prime}}$ such that $\# N=\# N^{\prime}, N$ and $N^{\prime}$ are disjoint and for all $i \in N$ there is a unique $i^{\prime} \in N^{\prime}$ such that $p(i)=p^{\prime}\left(i^{\prime}\right)$. Since $M(p)=M\left(p^{\prime}\right)$ it follows that $\varphi(p)=\varphi\left(p^{\prime}\right)$. So, consistency implies that $\varphi(p)=\varphi\left(p^{\prime}\right)=\varphi\left(p, p^{\prime}\right)$. Therefore with a little abuse of notation we will write profile $(p, p)$ instead of $\left(p, p^{\prime}\right)$.

Lemma 11 Let $p$ and $q$ be profiles such that $D(p)=k D(q)$ for some integer $k \geq 1$. Then $\varphi(p)=$ $\varphi(q)$ and $\varphi_{\text {Kemeny }}(p)=\varphi_{\text {Kemeny }}(q)$.

Proof. We only prove $\varphi(p)=\varphi(q)$. The proof of the second equation follows similarly from the properties: Pareto optimality, neutrality, consistency, monotonicity, and pairwiseness. Joining almost maximal conflict profiles for each pair $(a, b)$ with $D(p)_{a b}>0$ and possibly adding maximal conflicts yields a profile $r$ such that $M(r)=2 M(p)+2 s \widehat{E}=2 k M(q)+2 t \widehat{E}$ for some positive integers $s$ and $t$, where $\widehat{E}$ is the $m \times m$ matrix with all cells equal to one except $\widehat{E}_{a a}=0$ for all $a^{4}$. Replicating $p$ once and $q$ for $2 k$ times yields profiles $p^{\prime}=(p, p)$ and $q^{\prime}=(q, q, \ldots, q)$ such that $M(r)=M\left(p^{\prime}\right)+2 s \widehat{E}$ and $M(r)=M\left(q^{\prime}\right)+2 t \widehat{E}$. Note that maximal conflicts have the pairwise matrix equal to an even multiple of $\widehat{E}$. Therefore pairwiseness, consistency and Lemma 9 imply that

\footnotetext{
${ }^{4}$ Note that $\widehat{E}$ can be considered as the pairwise matrix of any maximal conflict. Furthermore, for each unit of $D(p)_{a b}$, one almost maximal conflict, $\pi(1, a b)$ is added to build $r$. As $M(\pi(1, a b))_{a b}=2$ we have $M(r)=$ $2 M(p)+2 s \widehat{E}$.
} 
$\varphi\left(p^{\prime}\right)=\varphi(r)$ and $\varphi\left(q^{\prime}\right)=\varphi(r)$. Furthermore consistency implies $\varphi\left(p^{\prime}\right)=\varphi(p)$ and $\varphi\left(q^{\prime}\right)=\varphi(q)$. So, the desired result $\varphi(p)=\varphi(q)$ follows.

Remark 6 Note that Lemmas 9 and 10 follow immediately from the Condorcet condition as stated in Young and Levenglick (1978). This condition in our notation means that for all profiles $p$ and for all alternatives a such that $D(p)_{a x} \geq 0$ for all alternatives $x$ different from a (hence alternative $a$ is a weak Condorcet-winner at $p$ ):

1. If $D(p)_{a y}=0$ for some alternative $y$ different from a, then $R \in \varphi(p) \Leftrightarrow R^{\prime} \in \varphi(p)$ for all $R$ and $R^{\prime}$ in $\mathbb{L}$ forming an elementary change in ay.

2. If $D(p)_{a y}>0$ for some alternative $y$ different from $a$, then $R \notin \varphi(p)$ for $R \in \mathbb{L}$ with .ya. $=R$.

Although under different assumptions, Lemma 11 is also deduced as an intermediate result in Young and Levenglick (1978, Lemma 1).

The following step is to prove that $\varphi$ and $\varphi_{\text {Kemeny }}$ coincide on profiles where the pairwise majority relation is non cyclic. Roughly speaking, this boils down to proving that $\varphi$ is Condorcet like.

Lemma 12 Let $p$ be a profile in $\mathbb{L}^{N}$ and $a_{1}, a_{2}, \ldots, a_{m}$ a numbering of the alternatives such that for all $1 \leq i<j \leq m$

$$
M(p)_{a_{i} a_{j}} \geq M(p)_{a_{j} a_{i}} .
$$

Then $\varphi_{\text {Kemeny }}(p)=\cap\left\{\mathbb{L}_{a_{i} a_{j}}: 1 \leq i<j \leq m\right.$ such that $\left.M(p)_{a_{i} a_{j}}>M(p)_{a_{j} a_{i}}\right\}=\varphi(p)$.

Proof. Note that the intersection at the right hand side is not empty so the right equality follows because of Lemma 10, Lemma 11 and consistency. Now $\varphi_{\text {Kemeny }}$ is consistent. This and Lemma 10 and Lemma 11 yields the left equality.

Lemma $13 \varphi$ is strongly monotone. Moreover, for all non-empty finite subsets $N$ of $\mathcal{N}$, all agents $i$ in $N$, all alternatives $a$ and $b$ in $A$ and all profiles $p$ and $q$ in $\mathbb{L}^{N}$, such that $\mathbb{L}_{a b} \cap \varphi(p) \neq \emptyset$, $(b, a) \in p(i),(a, b) \in q(i), \delta(p(i), q(i))=1$ and $\left.p\right|_{N-\{i\}}=\left.q\right|_{N-\{i\}}$,

$$
\varphi(q)=\mathbb{L}_{a b} \cap \varphi(p) .
$$

Proof. Let $N, i, a, b, p$ and $q$ as in the formulation of the Lemma. Without loss of generalization suppose that $\{1,2\} \cap N=\emptyset$. Consider $r \in \mathbb{L}^{\{1,2\}}$ such that $r=\pi(1, a b)$. Lemma 10 yields that $\varphi(r)=\mathbb{L}_{a b}$. Also we have $D(q)=D(p, r)$. So, as $\mathbb{L}_{a b} \cap \varphi(p) \neq \emptyset$ Lemma 11 and consistency imply $\varphi(q)=\varphi(p, r)=\varphi(r) \cap \varphi(p)=\mathbb{L}_{a b} \cap \varphi(p)$.

Now we are able to prove the characterization of Kemeny rule.

Theorem 2 The Kemeny rule is the only correspondence which is simultaneously Pareto optimal, neutral, pairwise, consistent and monotone.

Proof. (If part) Lemma 7 and 8 show that the Kemeny rule satisfies these five conditions.

(Only if part) Let $\varphi$ be a Pareto optimal, neutral, pairwise, consistent and monotone rule. Let $p$ be a profile. For $\lambda(p, R)=\sum_{a b \notin R} D(p)_{a b}$, let $\lambda(p, \mathbb{L})=\min \{\lambda(p, R): R \in \mathbb{L}\}$. In the sequel we will prove by induction on $\lambda(p, \mathbb{L})$ that $\varphi(p)=\varphi_{\text {Kemeny }}(p)$. In view of Lemma 11 we may assume that $p$ consists of almost maximal conflicts only. 
(Induction basis) Let $\lambda(p, \mathbb{L})=0$. In that case there exist linear orders $R$ such that $D(p)_{a b}=0$ for all $a b \notin R$. So there are no pairwise majority cycles at $p$. Hence, by Lemma $12 \varphi(p)=$ $\varphi_{\text {Kemeny }}(p)$.

(Induction step) Let $\lambda(p, \mathbb{L})=k+1$. Define the set of unanimous pairs at $p$ by $U(p)=\{(x, y) \in$ $A \times A:$ :x.y. $=p(i)$ for all agents $i \in N\}$.

Claim Let $(a, b) \notin U(p)$ and $\mathbb{L}_{a b} \cap \varphi(p) \neq \emptyset$ and $\mathbb{L}_{a b} \cap \varphi_{\text {Kemeny }}(p) \neq \emptyset$. Then $\mathbb{L}_{a b} \cap \varphi(p)=$ $\mathbb{L}_{a b} \cap \varphi_{\text {Kemeny }}(p)$.

Proof of the claim Let $R^{K} \in \mathbb{L}_{a b} \cap \varphi_{\text {Kemeny }}(p)$ and $R \in \mathbb{L}_{a b} \cap \varphi(p)$. Then it follows by the definition of $\varphi_{\text {Kemeny }}$ that $\lambda\left(p, R^{K}\right)=k+1$. As $p$ consists of almost maximal conflicts we may assume that there exists an update say $q$ of $p$ towards $R$ and $R^{K}$ such that:

$$
\begin{aligned}
q(i) & =(p(i)-\{(b, a)\}) \cup\{(a, b)\} \text { for some } i \in N \\
q(j) & =p(j) \text { for all } j \in N-\{i\} .
\end{aligned}
$$

Lemma 13 implies that $\mathbb{L}_{a b} \cap \varphi_{\text {Kemeny }}(p)=\varphi_{\text {Kemeny }}(q)$ and $\mathbb{L}_{a b} \cap \varphi(p)=\varphi(q)$. As $\lambda\left(q, R^{K}\right)=k$, the induction hypothesis implies $\varphi_{\text {Kemeny }}(q)=\varphi(q)$. Hence, $\mathbb{L}_{a b} \cap \varphi(p)=\mathbb{L}_{a b} \cap \varphi_{\text {Kemeny }}(p)$.

\section{End of proof of claim}

Next we distinguish two cases:

Case: $\# \varphi(p) \geq 2$ and $\# \varphi_{\text {Kemeny }}(p) \geq 2$. Since $\# \varphi(p) \geq 2$ there are alternatives $a$ and $b$ such that $(a, b) \notin U(p)$ and $\mathbb{L}_{a b} \cap \varphi(p) \neq \emptyset, \mathbb{L}_{b a} \cap \varphi(p) \neq \emptyset$ and $\mathbb{L}_{a b} \cap \varphi_{\text {Kemeny }}(p) \neq \emptyset$. Hence, by the previous claim $\mathbb{L}_{a b} \cap \varphi(p)=\mathbb{L}_{a b} \cap \varphi_{\text {Kemeny }}(p)$. In case $\mathbb{L}_{b a} \cap \varphi_{\text {Kemeny }}(p) \neq \emptyset$ the previous claim implies that also $\mathbb{L}_{b a} \cap \varphi(p)=\mathbb{L}_{b a} \cap \varphi_{\text {Kemeny }}(p)$. So, then $\varphi(p)=\varphi_{\text {Kemeny }}(p)$. If $\mathbb{L}_{b a} \cap \varphi_{\text {Kemeny }}(p)=$ $\emptyset$, then $\varphi_{\text {Kemeny }}(p) \subseteq \varphi(p)$. So, \# $\varphi(p) \geq 2$ implies $\varphi_{\text {Kemeny }}(p) \subseteq \varphi(p)$. Similarly \# $\varphi_{\text {Kemeny }}(p) \geq 2$ implies $\varphi_{\text {Kemeny }}(p) \supseteq \varphi(p)$. Hence, in this case $\varphi_{\text {Kemeny }}(p)=\varphi(p)$.

Case: There is a renaming $\varphi_{1}$ and $\varphi_{2}$ of $\varphi$ and $\varphi_{\text {Kemeny }}$ and there are linear orders $R^{1}$ and $R^{2}$ such that $\varphi_{1}(p)=\left\{R^{1}\right\}$ and $R^{2} \in \varphi_{2}(p)$. Note that we are done if $\varphi_{2}(p)=\left\{R^{1}\right\}$. So, therefore to the contrary suppose that $R^{2} \neq R^{1}$. We end the proof by showing that this assumption leads to a contradiction.

Pareto optimality implies that $U(p) \subseteq R^{1} \cap R^{2}$. Next we prove that $R^{1} \cap R^{2} \subseteq U(p)$. Suppose to the contrary that $\left(R^{1} \cap R^{2}\right)-U(p) \neq \emptyset$ that is for some pair of alternatives $(a, b) \in\left(R^{1} \cap R^{2}\right)-U(p)$. Then, by the previous claim, it follows that $\mathbb{L}_{a b} \cap \varphi_{1}(p)=\mathbb{L}_{a b} \cap \varphi_{2}(p)$. Since $(a, b) \in R^{2}$ and $(a, b) \in R^{1}$, we have that $R^{2} \in \mathbb{L}_{a b} \cap \varphi_{2}(p)=\mathbb{L}_{a b} \cap \varphi_{1}(p)=\left\{R^{1}\right\}$. This would yield the contradiction $R^{2}=R^{1}$. Therefore, we may assume that $\left(R^{1} \cap R^{2}\right)-U(p)=\emptyset$ and as $R^{1} \cap R^{2} \subseteq U(p)$ we have $\left(R^{1} \cap R^{2}\right)=U(p)$.

For numbers $i$ and $j$ such that $\{i, j\}=\{1,2\}$ and different alternatives $x$ and $y$ we call the ordered pair $(x, y)$ "free" at $R^{i}$ if $. x y .=R^{i}$ and $. y . x .=R^{j}$. So, $x$ and $y$ are consecutively ordered $x$ above $y$ at $R^{i}$ and reversely ordered $y$ above $x$ at $R^{j}$. Therefore the pair $(x, y)$ is in $U(p)$. Furthermore, let $N_{x y}=\{i \in N:$. x.y. $=p(i)\}$ and let $n_{x y}=\# N_{x y}$. Because of $R^{1} \neq R^{2}$ we may choose alternatives $a$ and $b$ such that $(a, b)$ is free at $R^{1}$ and for all $(x, y)$ free at $R^{1}$, we either have $x=a$ or $. a . x .=R^{1}$. That is $(a, b)$ is the highest ordered free pair in $R^{1}$. As $(a, b)$ is free at $R^{1}$, we have $. b . a .=R^{2}$. Since .b.a. $=R^{2}$ and $. a . b .=R^{1}$, we may take $(y, x)$ free in $R^{2}$, such that $b=y$ or .b.y. $=R^{2}, x=a$ or $. x . a .=R^{2}$ and for all free $(c, d)$ in $R^{2}$ if $d \neq x$, then either $. d . x .=R^{2}$ or .a.d. $=R^{2}$. So, $(y, x)$ is the lowest free pair in $R^{2}$ just above $a$ in $R^{2}$. Where we may find this pair ordered between $b$ and $a$ in $R^{2}$.

Now if $y=a$ or $. y . a . b .=R^{1}$, then there is a free pair between $x$ and $y$ in $R^{1}$ and as $. x . y .=R^{1}$ this violates the assumption that $(a, b)$ is the highest ordered free pair at $R^{1}$. So, $b=y$ or.$b \cdot y$. $=R^{1}$, that is $b$ is weakly preferred to $y$ at $R^{1}$. If $x=b$ or $. a b \cdot x .=R^{1}$, then there is a free pair between 
$x$ and $a$ at $R^{2}$ contradicting that $(y, x)$ is the lowest free pair in $R^{j}$ just before $a$ in $R^{j}$. Therefore $x=a$ or $x . a b .=R^{1}$, which means that $x$ is weakly preferred to $a$ at $R^{1}$. Therefore at $R^{1}$, we have: $x$ is weakly preferred to $a$, and $a$ strictly to $b$, and $b$ weakly to $y$, whereas, by the choice of $(y, x)$, at $R^{2}$, we have: $b$ is weakly preferred to $y$, and $y$ strictly to $x$, and $x$ weakly to $a$. Now since $U(p)=\left(R^{1} \cap R^{2}\right)$, we have either $b=y$ or $N_{b y}=N$ and either $x=a$ or $N_{x a}=N$. Therefore $N_{a b} \subseteq N_{a y} \subseteq N_{x y}$ and $N_{y x} \subseteq N_{b x} \subseteq N_{b a}$. Consider $R_{b a}^{1}=\left(R^{1}-\{(a, b)\}\right) \cup\{(b, a)\}$ and profile $q$ such that $q(i)=R^{1}$ if $i \in N_{a b}$ and $q(i)=R_{b a}^{1}$ if $i \in N_{b a}$. Now strong monotonicity which follows from Lemma 13, the fact that $q(i)$ is between $p(i)$ and $R^{1}$ for all $i \in N$ and $\varphi_{1}(p)=\left\{R^{1}\right\}$ yield that $\varphi_{1}(q)=\left\{R^{1}\right\}$. Hence, by Remark 3 , it follows that $n_{a b}>n_{b a}$. Similarly by considering a preference $R_{x y}^{2}=\left(R^{2}-\{(y, x)\}\right) \cup\{(x, y)\}$ it follows that $n_{y x} \geq n_{x y}$. But then $N_{a b} \subseteq N_{a y} \subseteq N_{x y}, n_{y x} \geq n_{x y}$ and $N_{y x} \subseteq N_{b x} \subseteq N_{b a}$ imply $n_{a b} \leq n_{a y} \leq n_{x y} \leq n_{y x} \leq n_{b x} \leq n_{b a}$. Hence, we have the contradiction $n_{a b} \leq n_{b a}$, which ends the proof.

\section{$5 \quad$ Discussion and Further Research}

Update monotonicity, as also discussed in the introduction, is essentially a monotonicity condition for rules. We investigated preference correspondences and their reaction to an increase in support for a collective preference. Roughly speaking, we have analyzed monotone rules both in case the rules are convex valued and in case they are not. In the former, our finding is a class of rules that do not involve well-known convex valued rules such as scoring rules. In the latter, we end up with a new characterization of the Kemeny rule based on this monotonicity condition.

In following subsection we show, respectively, the independence of our conditions, some logical relations with the conditions in Young \& Levenglick characterization, some possible variations of update monotonicity and finally, we consider this condition in relation with single valuedness.

\subsection{Independence of Characterizing Conditions}

Below we provide some rules showing the independence of the characterizing conditions of Theorem 1 and 2.

Super majority rule $\left(\varphi_{g}\right)$ : Defined in Section 3 .

As shown in Theorem 1, $\varphi_{g}$ is Pareto optimal, neutral, convex valued, replication invariant, and strongly monotone. Rule $\varphi_{g}$ is pairwise because the $k$-majority-relation, on which it is solely based, is pairwise. To see that $\varphi_{g}$ is not consistent consider $A=\{a, b, c\}, p(1)=p(2)=p(3)=q(5)=a b c$ and $p(4)=p(6)=c b a$. Then $\varphi_{g}(p)=\{a b c\}$ and $\varphi_{g}(q)=\mathbb{L}$ in case $g(4)=3$. But $\varphi_{g}(p, q)=\mathbb{L}$ $\neq \varphi_{g}(p) \cap \varphi_{g}(q)$.

Biased super majority rule $\left(\widehat{\varphi}_{g}\right)$ Define the rule $\widehat{\varphi}_{g}$ for an arbitrary profile $p$ as follows: $\widehat{\varphi}_{g}(p)=\{R, \bar{R}\}$ whenever $p=\left(R^{S}, \bar{R}^{N-S}\right)$ for some non-empty and finite subsets $S, N$ of $\mathcal{N}$ such that $S \varsubsetneqq N$ and $R$ and $\bar{R}$ are in $\mathbb{L}$ forming an elementary change. In all other cases $\widehat{\varphi}_{g}(p)=\varphi_{g}(p)$.

Clearly, $\widehat{\varphi}_{g}$ is Pareto optimal, neutral, convex valued, replication invariant and monotone. It is not consistent because $\varphi_{g}$ is not consistent. Consider profiles $q=\left(R_{1}^{T}, R_{2}^{N-T}\right)$ for arbitrary preferences $R_{1}$ and $R_{2}$, such that $\# T \geq g(\# N)$. It follows that $\widehat{\varphi}_{g}$ is not strongly monotone, because for an elementary change $\bar{R}_{1}$ between $R_{1}$ and $R_{2}$, we have $\widehat{\varphi}_{g}\left(R_{1}^{T}, \bar{R}_{1}^{N-T}\right)=\left\{R_{1}, \bar{R}_{1}\right\}$ 
$\nsubseteq \widehat{\varphi}_{g}\left(R_{1}^{T}, R_{2}^{N-T}\right)=\left\{R_{1}\right\}$. This rule shows that the stronger monotonicity condition in Theorem 1 is logically essential.

Kemeny rule $\left(\varphi_{\text {Kemeny }}\right)$ : Defined in Section 4 .

As discussed in Section 4, $\varphi_{\text {Kemeny }}$ is Pareto optimal, neutral, pairwise, consistent and strongly monotone. By Remark 1, it is also replication invariant. The Kemeny rule, however, is not convex valued, e.g., for a Condorcet profile, it assigns the profile itself as Kemeny rankings.

Selective Kemeny rule $\left(\psi_{\text {Kemeny }}\right):$ A rule $\varphi$ is called a selective Kemeny rule whenever for some enumeration of all linear orders, for instance $\mathbb{L}=\left\{R_{1}, R_{2}, \ldots, R_{m !}\right\}, \quad \psi_{\text {Kemeny }}(p)=$ $\left\{R_{i}: i\right.$ is minimal for all $R_{i}$ in $\left.\varphi_{\text {Kemeny }}(p)\right\}$,

i.e., $\psi_{\text {Kemeny }}$ assigns to each profile the Kemeny ranking with the minimal predefined index.

Note that, since for any profile $p, \psi_{\text {Kemeny }}(p) \subseteq \varphi_{\text {Kemeny }}(p)$, selective Kemeny rule also satisfies Pareto optimality. It is also pairwise since for two profiles, say $p$ and $q$, with identical pairwise matrices, $\varphi_{\text {Kemeny }}(p)=\varphi_{\text {Kemeny }}(q)$ therefore $R_{i} \in \varphi_{\text {Kemeny }}(p)$ with minimal $i$ is the same as $R_{i} \in \varphi_{\text {Kemeny }}(q)$ with minimal $i$. To show that the rule is consistent let $p, q$ be two profiles such that $\psi_{\text {Kemeny }}(p) \cap \psi_{\text {Kemeny }}(q)=\left\{R_{j}\right\}$. Then $j$ is the smallest number among $R_{i}$ in both $\varphi_{\text {Kemeny }}(p)$ and $\varphi_{\text {Kemeny }}(q)$. Hence, by consistency, $R_{j}$ is in $\varphi_{\text {Kemeny }}(p, q)$ and $R_{j}$ has the smallest index among those $R_{i}$ which are in $\varphi_{\text {Kemeny }}(p) \cap \varphi_{\text {Kemeny }}(q)=\varphi_{\text {Kemeny }}(p, q)$. So, $\psi_{\text {Kemeny }}(p, q)=\left\{R_{j}\right\}$. Monotonicity is straightforward by strong monotonicity of Kemeny rule. It is also trivially convex valued as it always assigns a single outcome to each profile, yet it is not neutral by construction.

Trivial rule $\left(\varphi_{T}\right)$ : A rule $\varphi$ is called the trivial rule if for all profiles $p$ in $\mathbb{L}^{N}, \varphi(p)=\mathbb{L}$.

By construction, the trivial rule always assign the same set of linear orders, $\mathbb{L}$, therefore it is neutral, pairwise, convex valued, consistent, replication invariant, and monotone. Obviously it is not Pareto optimal.

Dictatorial rule $\left(\varphi_{\text {dictatorial }}\right):$ A rule $\varphi$ is called a dictatorial rule if there exists an individual $d \in N$ such that for all profiles $p, \varphi(p)=\{p(d)\}$.

Dictatorial rule $\varphi_{\text {dictatorial }}$ is known to be Pareto optimal. To show consistency, for any predefined order over individuals in $\mathcal{N}$, to choose the dictator for each subset of individuals $N \subseteq \mathcal{N}$, consider two profiles and two dictators in each society. If two profiles agree on an outcome, when the two profiles merge, one dictator will remain as the dictator, according to the predefined order, of the merged profile hence the outcome will be the same. It is also trivially convex valued as it always assigns a single outcome to each profile. It is neutral and monotone by construction. It is not anonymous; hence, it also fails to be pairwise and replication invariant.

Borda rule $\left(\varphi_{\text {Borda }}\right)$ : Defined in Example 1 of Section 2, where the score vector $\vec{s}=(m, m-$ $1, m-2, \ldots, 1)$.

Borda is known to be Pareto optimal, neutral, pairwise and consistent and therewith replication invariant. By construction it is convex valued like all score rules. It fails to be monotone; hence, it is also not strongly monotone.

The table below summarizes the findings discussed above. It shows the logical independence of the characterizing conditions in both Theorem 1 and Theorem 2. We denote $\varphi_{\text {Kemeny }}$ and $\psi_{\text {Kemeny }}$, by $\varphi_{K}$ and $\psi_{K}$ respectively. 


$\begin{array}{lccccccc} & \varphi_{g} & \varphi_{K} & \psi_{K} & \varphi_{T} & \varphi_{\text {dictatorial }} & \varphi_{\text {Borda }} & \widehat{\varphi}_{g} \\ \text { Pareto optimal } & \mathrm{Y} & \mathrm{Y} & \mathrm{Y} & \mathrm{N} & \mathrm{Y} & \mathrm{Y} & \mathrm{Y} \\ \text { Neutral } & \mathrm{Y} & \mathrm{Y} & \mathrm{N} & \mathrm{Y} & \mathrm{Y} & \mathrm{Y} & \mathrm{Y} \\ \text { Consistent } & \mathrm{N} & \mathrm{Y} & \mathrm{Y} & \mathrm{Y} & \mathrm{Y} & \mathrm{Y} & \mathrm{N} \\ \text { Convex valued } & \mathrm{Y} & \mathrm{N} & \mathrm{Y} & \mathrm{Y} & \mathrm{Y} & \mathrm{Y} & \mathrm{Y} \\ \text { Replication invariant } & \mathrm{Y} & \mathrm{Y} & \mathrm{Y} & \mathrm{Y} & \mathrm{N} & \mathrm{Y} & \mathrm{Y} \\ \text { Pairwise } & \mathrm{Y} & \mathrm{Y} & \mathrm{Y} & \mathrm{Y} & \mathrm{N} & \mathrm{Y} & \mathrm{Y} \\ \text { Monotone } & \mathrm{Y} & \mathrm{Y} & \mathrm{Y} & \mathrm{Y} & \mathrm{Y} & \mathrm{N} & \mathrm{Y} \\ \text { Strongly monotone } & \mathrm{Y} & \mathrm{Y} & \mathrm{Y} & \mathrm{Y} & \mathrm{Y} & \mathrm{N} & \mathrm{N}\end{array}$

\subsection{Logical Relations Regarding Young \& Levenglick Characterization of the Kemeny Rule}

In this subsection we show that the two sets of characterizing conditions of the Kemeny can be deduced from one another directly. First, in Lemma 14 and 15, it is proved that the conditions of Young and Levenglick imply the characterizing conditions of Theorem 2.

Lemma 14 Strong monotonicity is implied by neutrality, consistency and the Condorcet condition.

Proof. Let $\varphi$ be such a rule. Let $p$ and $q$ be profiles and $i$ an agent such that $\left.p\right|_{N-\{i\}}=\left.q\right|_{N-\{i\}}$ and $q(i)$ is an elementary change of $p(i)$ in $a b$. It is sufficient to prove that $\varphi(q)=\mathbb{L}_{a b} \cap \varphi(p)$ in case $\mathbb{L}_{a b} \cap \varphi(p) \neq \emptyset$. Let $r=(p, p, \pi(1, a b))$, then $D(r)=2 D(q)$. Also the Condorcet condition implies that $\varphi(\pi(1, a b))=\mathbb{L}_{a b}$. Consistency implies $\varphi(r)=\varphi(p) \cap \mathbb{L}_{a b}$ as $\mathbb{L}_{a b} \cap \varphi(p) \neq \emptyset$. In view of Remark 6, $\varphi(q)=\varphi(r)=\varphi(p) \cap \mathbb{L}_{a b}$.

Lemma 15 Pareto optimality is implied by neutrality, consistency and the Condorcet condition.

Proof. Let $\varphi$ be a neutral and consistent rule which satisfies the Condorcet condition. Let $p$ be a profile in $\mathbb{L}^{N}$ and let $a$ and $b$ be two different alternatives such that $. a . b .=p(i)$ for all agents $i$ in $N$. And, to the contrary, let $R \in \varphi(p)$ with $. b . a .=R$. First, we prove that in this case we may take $p$ such that $. a b .=p(i)$ for all agents $i$ in $N$. Let $. a x_{1} x_{2} \ldots x_{t} b .=p(j)$ for some agent $j$. Let $s$ be the smallest number such that $. x_{s} \cdot a .=R$ assuming that there are $x_{u}$ such that $. x_{u} \cdot a .=R$. Consider profile $q$ such that $. x_{s} a x_{1} x_{2} \ldots x_{s-1} x_{s+1} \ldots x_{t} b .=q(j)$ and $q(i)=p(i)$ for $i \in N-\{j\}$. Clearly $q(j) \subseteq p(j) \cup R$, hence by monotonicity, which holds by Lemma 14, we have that $R \in \varphi(q)$ where a.b. $=q(i)$ for all agents $i$ in $N$. In case for all $x_{u}$ we have that $. a . x_{u} .=R$. we may take $q(j)=. a x_{1} x_{2} \ldots x_{t-1} b x_{t}$. and have the same result. Therefore, by the finiteness of the set of alternatives, we may assume that $. a b .=p(i)$ for all agents $i$ in $N$. Next, consider the permutation $\sigma$ on $A$ such that $\sigma(a)=b, \sigma(b)=a$ and $\sigma(x)=x$ for all alternatives $x \in A-\{a, b\}$. Note that $\sigma p(i) \subseteq p(i) \cup R$ for all agents $i$ in $N$. Hence, similar as in Lemma 14 we find that $\varphi(\sigma p)=\varphi(p) \cap \mathbb{L}_{b a}$. Hence, $\varphi(\sigma p) \subseteq \mathbb{L}_{b a}$. But neutrality implies that $\varphi(\sigma p)=\sigma(\varphi(p))$ and as $R \in \varphi(p)$ this yields the contradiction that $\sigma R \in \varphi(\sigma p)$ that is $\varphi(\sigma p) \cap \mathbb{L}_{a b} \neq \emptyset$.

Note that Lemma 1 in Young and Levenglick (1978) shows that neutrality, consistency and the Condorcet condition implies pairwiseness. So, all together, the conditions of Young and Levenglick -neutrality, consistency and the Condorcet condition- imply the set of conditions: Pareto optimality, neutrality, pairwiseness, consistency and monotonicity. The latter set is the set of characterizing conditions used in Theorem 2. 
Next, we show that this latter set of conditions implies the former, which actually boils down to the following Lemma.

Lemma 16 Pareto optimality, neutrality, pairwiseness, consistency and monotonicity imply the Condorcet condition.

Proof. Let $\varphi$ be a Pareto optimal, neutral, consistent, pairwise and monotone rule. Let $p$ be a profile in $\mathbb{L}^{N}$. Let $c$ and $d$ be two different alternatives and $R$ in $\mathbb{L}$ such that $. c d .=R$ and $R \in \varphi(p)$.

Claim: There is a profile $q$, an update of $p$ towards $R$, such that $R \in \varphi(q), M(p)_{c d}=M(q)_{c d}$ and $. c d .=q(i)$ or $. d c .=q(i)$ for all agents $i$ in $N$.

Proof of Claim Take agent $j$ arbitrarily. We distinguish two cases.

Case $. c x_{1} x_{2} \ldots x_{t} d .=p(j)$. Define profile $r$ by $r(i)=p(i)$ for $i \in N-\{j\}$. In case there is a smallest number $s$ such that $. x_{s} . c d .=R$ defined $r(j)=. x_{s} c x_{1} x_{2} \ldots x_{s-1} x_{s+1} \ldots x_{t} d .$. If $R$ is such that $. c d . x_{s} .=R$ for all $s$ take $r(j)=. c x_{1} x_{2} \ldots x_{t-1} d x_{t} .$. Then $r$ is an update of $p$ towards $R$ such that $M(p)_{c d}=M(r)_{c d}, M(p)_{c d}=M(r)_{c d}$ and by monotonicity we have $R \in \varphi(r)$.

Case $. d x_{1} x_{2} \ldots x_{t} c .=p(j)$. Define profile $r$ by $r(i)=p(i)$ for $i \in N-\{j\}$. In case there is a smallest number $s$ such that $. x_{s} . c d .=R$ defined $r(j)=x_{s} d x_{1} x_{2} \ldots x_{s-1} x_{s+1} \ldots x_{t} c$. If $R$ is such that $. c d . x_{s} .=R$ for all $s$ take $r(j)=. d x_{1} x_{2} \ldots x_{t-1} c x_{t}$. . Then $r$ is an update of $p$ towards $R$ such that $M(p)_{c d}=M(r)_{c d}, M(p)_{c d}=M(r)_{c d}$ and by monotonicity we have $R \in \varphi(r)$.

Repeating these cases a finite number of times yields the desired result.

End of proof of Claim:

Let $a$ be an alternative such that $D(p)_{a x} \geq 0$ for all alternatives $x$ in $A-\{a\}$. In order to prove the second part of the Condorcet condition let $D(p)_{a y}=0$ for some alternative $y$ in $A-\{a\}$. Let $\{c, d\}=\{a, y\}$ and $. c d .=R$ for some linear order in $\varphi(p)$. We have to prove that $R^{\prime}=. d c$. which forms with $R$ an elementary change in $c$ and $d$ is in $\varphi(p)$.Consider the permutation $\sigma$ on $A$ such that $\sigma(a)=y, \sigma(y)=a$ and $\sigma(z)=z$ for all $z \in A-\{a, y\}$. Take $q$ as in the foregoing claim. Then $D(q)_{a y}=D(q)_{y a}=0$ and strong monotonicity (which follows from Lemma 13) implies $R \in \varphi(q) \subseteq \varphi(p)$. Neutrality now implies $R^{\prime} \in \varphi(\sigma q)$. But as for all agents $i$ either $. a y .=q(i)$ or $. y a .=q(i)$ and $D(q)_{a y}=D(q)_{y a}=0$ it follows that $M(q)=M(\sigma q)$. Hence, pairwiseness implies $R^{\prime} \in \varphi(q) \subseteq \varphi(p)$, which proves the second part of the Condorcet condition.

In order to prove the first part of the Condorcet condition let $D(p)_{a y}>0$ and let to the contrary $. y a .=R^{\prime \prime}$ be in $\varphi(p)$. Consider a profile $r$ which consists of two times $p$ and almost maximal conflict profile $\pi\left(D(p)_{a y}, y a\right)$.So, $r=\left(p, p, \pi\left(D(p)_{a y}, y a\right)\right)$. Then consistency and Lemma 10 imply $\varphi(r)=\varphi(p) \cap \mathbb{L}_{y a}$. Hence, $R^{\prime \prime} \in \varphi(r) \subseteq \mathbb{L}_{y a}$. But $D(r)_{a x}=2 D(p)_{a x} \geq 0$ for all $x \in A-\{a, y\}$ and $D(r)_{a y}=D(r)_{y a}=0$. Hence, as $R^{\prime \prime} \in \varphi(r)$ the second part of the Condorcet condition implies $\varphi(r) \cap \mathbb{L}_{a y} \neq \emptyset$ which contradicts $\varphi(r) \subseteq \mathbb{L}_{y a}$.

\subsection{Possible Variations in the Concept of Update and Monotonicity}

By different variations in the concept of an update, one may acquire various conditions in a similar manner. We define Kemeny-update monotonicity as the ability of a rule to preserve the collective preferences whenever the preference profile is updated towards the collective preference in terms of a decrease in Kemeny ${ }^{5}$ distance of each individual preference to the collective preference. Similarly, extreme-update monotonicity requires that a rule preserves the collective preference whenever some

\footnotetext{
${ }^{5}$ Kemeny distance counts the number of ordered pairs on which two binary relations i.e., strict preferences, are different. Note that one may formulate distance based conformity with respect to other metrics, however for our model we restrict our attention to Kemeny metric.
} 
(possibly all) individuals identically imitate the same collective preference while others remain unchanged. Note that Kemeny-update monotonicity is the strongest of all variations mentioned whereas extreme-update monotonicity is the weakest.

Kemeny-update monotonicity: A rule is (strongly) Kemeny-update monotone if for all profiles $p \in \mathbb{L}^{N}$, and for all $R \in \varphi(p)$, and for all $q \in \mathbb{L}^{N}$ such that $\delta(q(i), R) \leq \delta(p(i), R)$ for all $i \in N$,

$$
\begin{aligned}
R & \in \varphi(q) \\
\text { (and in addition } \varphi(q) & \subseteq \varphi(p) \text { ). }
\end{aligned}
$$

Note that in the regular update monotonicity condition, the betweenness requirement, already implied a decrease in the distance between the individual preference and the outcome. Dropping this leads to a strenghtening of the monotonicity condition to the extent that even the Kemeny rule is not Kemeny-update monotone. In Can and Storcken (2011), it is shown that strong Kemeny-update monotonicity leads to so called impossibility theorems.

One can also only consider transformations of profiles in which some agents identically copy the outcome and the rest remain the same. In such extreme updates of collective preference, we have a much more weaker update monotonicity condition:

Extreme-update monotonicity: A rule is extreme-update monotone if for all profiles $p \in \mathbb{L}^{N}$, and for all $R \in \varphi(p)$, and for all $q \in \mathbb{L}^{N}$ such that $q(i) \in\{R, p(i)\}$ for all $i \in N$,

$$
R \in \varphi(q) .
$$

Extreme-update monotonicity is the least demanding of all since it requires that a rule preserves the outcome only when some agents copy the outcome while the rest remains as they are. In that context, it resembles the simple monotonicity condition in collective choice rules. However weak extreme-update monotonicity may be; most rules, including Borda and other score rules, still fail to satisfy it. For instance, let $A=\{a, b, c\}$, and $n=4$ and consider a profile $p$, such that individuals respectively have the following preferences: $a b c, b a c, a c b, c a b$. Consider the Borda rule, $\varphi_{\text {Borda }}(p)=\{a b c, a c b\}$. Consider, now, the extreme update $q$ of $p$ towards $a c b$, where individuals respectively have, $a b c, b a c, a c b, a c b$, i.e. $4^{t h}$ individual imitated one of the outcomes. It is easy to see, however, that $\varphi(q)=\{a b c\}$ is the unique collective preference at the updated profile.

\subsection{Update Monotone Welfare Functions}

In this subsection we consider single valued rules, which are also known as welfare functions. Because a singleton set is convex, such rules are convex valued. Furthermore, it is easy to see that monotone single valued rules are strongly monotone. The selective Kemeny rule is an example of a non trivial monotone welfare function. Similarly, selective super majority rules $\psi_{g}$ can be defined by assigning to a profile $p$ that order in $\varphi_{g}(p)$ having the smallest index. It is straightforward to prove that selective super majority rules are Pareto optimal, replication invariant, pairwise and (strongly) monotone. They violate, however, the conditions of neutrality and consistency. The condition of neutrality is also violated by selective Kemeny rules. In view of Lemma 4, it is straightforward that rules satisfying the conditions in Theorem 1 cannot be single valued. Similarly, welfare functions that are Pareto optimal, neutral and monotone cannot be replication invariant. We leave the study of welfare functions satisfying those conditions, except replication invariance, for future research.

As hinted in the discussion, there is a strong logical relationship between strategy-proofness and monotonicity conditions in the choice rules framework. Bossert and Storcken (1992) analyze 
strategy-proofness for single valued preference correspondences with the following result. Let $A$ contain at least 4 alternatives and fix $N$, such that $\# N$ is even: then, there does not exist a welfare function which is simutaneously, nonimposed, coalitional strategy-proof and weakly extrema independent. Nonimposition, which is in fact implied by Pareto optimality, means that the function is surjective, i.e. the range of the welfare function is the complete set of linear order. Weak extrema independence is formally introduced below:

Definition 3 A welfare function $\psi$ is weakly extrema independent if for any two profiles $p$ and $q$ orders $R_{1}, R_{2}, \widehat{R}_{1}$ and $\widehat{R}_{2}$ and disjoint coalitions $S$ and $T$ such that:

$$
\begin{aligned}
\# S & =\# T \\
p(i) & =R_{1}, q(i)=\widehat{R}_{1} \text { for } i \in S \\
p(i) & =R_{2}, q(i)=\widehat{R}_{2} \text { for } i \in T \\
p(i) & =q(i) \notin\left\{R_{1}, R_{2}, \widehat{R}_{1}, \widehat{R}_{2}\right\} \text { for } i \in N-(S \cup T), \\
p(N), q(N) & \subseteq \mathbb{L}_{R_{1} \cap R_{2}}=\mathbb{L}_{\widehat{R}_{1} \cap \widehat{R}_{2}} .
\end{aligned}
$$

we have $\psi(p)=\psi(q)$.

Proposition 3 Selective Kemeny rules are weakly extrema independent.

Proof. It is sufficient to prove that for two profiles as defined in Definition $3, \varphi_{\text {Kemeny }}(p)=$ $\varphi_{\text {Kemeny }}(q)$. Since Kemeny rule is pairwise it is sufficient to show that $M(p)=M(q)$. Note that $\mathbb{L}_{R_{1} \cap R_{2}}=\mathbb{L}_{\widehat{R}_{1} \cap \widehat{R}_{2}}$ is equivalent to $R_{1} \cap R_{2}=\widehat{R}_{1} \cap \widehat{R}_{2}$. Furthermore, as $\left.p\right|_{(S \cup T)}=\left(R_{1}^{S}, R_{2}^{T}\right)$ and $\left.q\right|_{(S \cup T)}=\left(\widehat{R}_{1}^{S}, \widehat{R}_{2}^{T}\right), R_{1} \cap R_{2}=\widehat{R}_{1} \cap \widehat{R}_{2}$ and $\# S=\# T$ it follows, although a bit cumbersome, that $M\left(\left.p\right|_{(S \cup T)}\right)=M\left(\left.q\right|_{(S \cup T)}\right)$. Of course $M\left(\left.p\right|_{N-(S \cup T)}\right)=M\left(\left.q\right|_{N-(S \cup T)}\right)$. So, $M(p)=M\left(\left.p\right|_{(S \cup T)}\right)+$ $M\left(\left.p\right|_{N-(S \cup T)}\right)=M\left(\left.q\right|_{(S \cup T)}\right)+M\left(\left.q\right|_{N-(S \cup T)}\right)=M(q)$.

Remark 7 Note that the condition $\# S=\# T$ is essential in proving that selective Kemeny rules are pairwise. Indeed the more demanding condition of extrema independence, at which this equality is not required, is not implied by pairwiseness. Selective Kemeny rules are not extrema independent which can be deduced by considering for instance maximal conflicts $p=\left(R_{1}^{S},-R_{1}^{N-S}\right)$ and $q=$ $\left(\widehat{R}_{1}^{S},-\widehat{R}_{1}^{N-S}\right)$ where $\# S>\# N-S$. Then $\psi_{\text {Kemeny }}(p)=\varphi_{\text {Kemeny }}(p)=\left\{R_{1}\right\}$ and $\psi_{\text {Kemeny }}(q)=$ $\varphi_{\text {Kemeny }}(q)=\left\{\widehat{R}_{1}\right\}$.

As selective Kemeny rules are Pareto, hence nonimposed, and weakly extreme independent, it follows that they are not coalitional strategy-proof. Indeed consider $N=\{1,2\}, A=\{a, b, c\}$ and profiles $p, q$ such that $p(1)=R_{6}=b a c, p(2)=q(2)=R_{4}=c b a, q(1)=R_{1}=a b c$ (although redundant for this example may take further $R_{2}=a c b, R_{3}=c a b$ and $R_{4}=c b a$ ). Then based on the indexation of the linear orders $\psi_{\text {Kemeny }}(p)=\left\{R_{4}\right\}$ and $\psi_{\text {Kemeny }}(q)=\left\{R_{1}\right\}$. As $R_{1}$ is strictly closer to $p(1)$ than $R_{4}$ it follows that $\psi_{\text {Kemeny }}$ is not strategy-proof and therewith not coalitional strategy-proof. Similarly, selective super majority rules $\psi_{g}$ are weakly extrema independent, non strategy-proof and not extrema independent.

Most probably, under some kind of non-bossiness condition, strategy-proofness implies update monotonicity. We stop here, however, as we hope that the above has convinced the reader that this subject is at least for the time being interesting enough to investigate further. 


\section{Appendix}

The following Proposition shows that intersections of subsets, being sufficiently large, are not empty.

Proposition 4 Let $T^{1}$ up to $T^{l}$ be a collection of $l$ subsets of finite and non-empty set $N$ such that $\# T^{j}>(l-1) \cdot \# N / l$. Then $\cap\left\{T^{j}: j \in\{1, \ldots, l\}\right\} \neq \emptyset$.

Proof. To prove the contra position suppose $\cap\left\{T^{j}: j \in\{1, \ldots, l\}\right\}=\emptyset$. Now we may take $T^{j}$ such that for all $i$ in $N$ there are precisely $l-1$ sets say $T^{i_{1}}$ up to $T^{i_{l-1}}$ such that $i$ is in each of these. So, as

$$
\sum_{j=1}^{l} \# T^{j}=\sum_{i \in N} \#\left\{T^{j}: i \in T^{j}\right\}
$$

it follows that $\sum_{j=1}^{l} \# T^{j}=\# N \cdot(l-1)$. Let $\# T^{1} \leq \# T^{j}$ for all $j \in\{1,2, \ldots, l\}$. Then $\# T^{1} \cdot l \leq$ $\sum_{j=1}^{l} \# T^{j}=\# N \cdot(l-1)$. But then $\# T^{1} \leq(l-1) \cdot \# N / l$, which proves the contra position.

Next we discuss a result on linear orders concerning the Kemeny distance.

Proposition 5 Let $R$ and $R^{a b}$ be two linear orders in $\mathbb{L}_{a b}$. Let $R^{b a}=\sigma_{a b} R^{a b}$, where $\sigma_{a b}$ is the permutation on $A$ such that $\sigma_{a b}(a)=b, \sigma_{a b}(b)=a$ and $\sigma_{a b}(c)=c$ for all $c \in A-\{a, b\}$. Then $\delta\left(R, R^{a b}\right)<\delta\left(R, R^{b a}\right)$.

Proof. Let $\bar{R}^{b a}, \bar{R}^{a b} \in \mathbb{L}$ be two linear orders that are between $R^{b a}$ and $R$ such that $\bar{R}^{b a}$ and $\bar{R}^{a b}$ form an elementary change in $b a$. Then it is straightforward to see that $\delta\left(\bar{R}^{b a}, R^{b a}\right)=\delta\left(\bar{R}^{a b}, R^{a b}\right)$. Furthermore by betweenness, $\delta\left(R, R^{b a}\right)=\delta\left(R, \bar{R}^{a b}\right)+\delta\left(\bar{R}^{a b}, \bar{R}^{b a}\right)+\delta\left(\bar{R}^{b a}, R^{b a}\right)$. By triangular inequality, $\delta\left(R, R^{a b}\right) \leq \delta\left(R, \bar{R}^{a b}\right)+\delta\left(\bar{R}^{a b}, R^{a b}\right)=\delta\left(R, \bar{R}^{a b}\right)+\delta\left(\bar{R}^{b a}, R^{b a}\right)$. Hence $\delta\left(R, R^{a b}\right)<$ $\delta\left(R, R^{b a}\right)$.

\section{References}

Bogart, K. (1973). Preference structures I: Distances between transitive preference relations. Journal of Mathematical Sociology 3(1), 49-67.

Borda, J. (1784). Memoire sur les elections an scrutin/histoire de l'academie des sciences pour 1781.

Bossert, W. and T. Storcken (1992). Strategy-proofness of social welfare functions: The use of the kemeny distance between preference orderings. Social Choice and Welfare 9(4), 345-360.

Can, B. and T. Storcken (2011). Impossibilities with Kemeny Updating. mimeo.

Copeland, A. (1951). A reasonable social welfare function. Technical report, mimeo, University of Michigan.

Kemeny, J. (1959). Mathematics without numbers. Daedalus 88(4), 577-591. 
Kemeny, J. and J. Snell (1962). Mathematical models in the social sciences. Boston: Ginn.

Muller, E. and M. Satterthwaite (1977). The equivalence of strong positive association and strategyproofness. Journal of Economic Theory 14(2), 412-418.

Storcken, T. (2008). Collective choice rules on convex restricted domains. Maastricht: METEOR Research Memoranda RM/08/003.

Young, H. (1974). An axiomatization of borda's rule. Journal of Economic Theory 9(1), 43-52.

Young, H. and A. Levenglick (1978). A consistent extension of condorcet's election principle. SIAM Journal on Applied Mathematics 35(2), 285-300. 